\title{
Isogeometric analysis of laminated composite and sandwich plates using a new inverse trigonometric shear deformation theory
}

ARTICLE in EUROPEAN JOURNAL OF MECHANICS - A/SOLIDS · JANUARY 2014

Impact Factor: 1.9 · DOI: 10.1016/j.euromechsol.2013.09.001

CITATIONS

17

5 AUTHORS, INCLUDING:

Chien thai Hoang

Ton Duc Thang University

30 PUBLICATIONS 383 CITATIONS

SEE PROFILE
DOWNLOADS

138
VIEWS

128

\section{Stéphane Pierre Alain Bordas}

University of Luxembourg

181 PUBLICATIONS 2,835 CITATIONS

SEE PROFILE 


\section{Accepted Manuscript}

Isogeometric analysis of laminated composite and sandwich plates using a new inverse trigonometric shear deformation theory

Chien H. Thai, A.J.M. Ferreira, S.P.A. Bordas, T. Rabczuk, H. Nguyen-Xuan

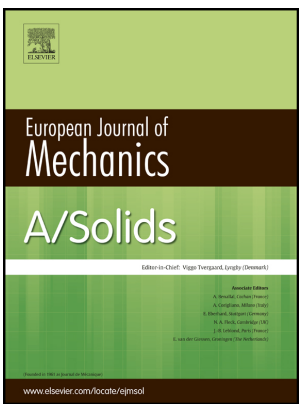

PII:

S0997-7538(13)00093-4

DOI:

10.1016/j.euromechsol.2013.09.001

Reference: EJMSOL 2970

To appear in: European Journal of Mechanics / A Solids

Received Date: 24 June 2013

Accepted Date: 3 September 2013

Please cite this article as: Thai, C.H., Ferreira, A.J.M., Bordas, S.P.A., Rabczuk, T., NguyenXuan, $\mathrm{H}$., Isogeometric analysis of laminated composite and sandwich plates using a new inverse trigonometric shear deformation theory, European Journal of Mechanics / A Solids (2013), doi: 10.1016/ j.euromechsol.2013.09.001.

This is a PDF file of an unedited manuscript that has been accepted for publication. As a service to our customers we are providing this early version of the manuscript. The manuscript will undergo copyediting, typesetting, and review of the resulting proof before it is published in its final form. Please note that during the production process errors may be discovered which could affect the content, and all legal disclaimers that apply to the journal pertain. 


\section{Highlights}

- We propose a new inverse tangent shear deformation theory (ITSDT) for laminated composite-material plates.

- The method does not require any shear correction factors due to using high-order deformation plate theory (HSDT).

- Static, free vibration and buckling plate models based on ITSDT are numerically solved using an isogeometric analysis (IGA)

- The proposed formulation requires C1-continuity generalized displacements and hence basis functions used in IGA fulfill this requirement.

- Intensive numerical studies have been conducted to show excellent performance of the present method. 


\title{
Isogeometric analysis of laminated composite and sandwich plates using a new inverse trigonometric shear deformation theory
}

\author{
Chien H. Thai ${ }^{a}$, S.P.A. Bordas ${ }^{b}$, A.J.M. Ferreira ${ }^{c}$, T. Rabczuk ${ }^{\text {d }}$, H. Nguyen-Xuan ${ }^{\mathrm{a}, e, *}$ \\ ${ }^{a}$ Division of Computational Mechanics, Ton Duc Thang University, HoChiMinh City, Vietnam \\ ${ }^{b}$ Institute of Mechanics and Advanced Materials, School of Engineering, College of Physical Sciences, Cardiff \\ University, Wales, UK \\ ${ }^{c}$ Departamento de Engenharia Mecanica, Faculdade de Engenharia, Universidade do Porto, Rua Dr. Roberto Frias, \\ 4200-465 Porto, Portugal \\ ${ }^{d}$ Institute of Structural Mechanics, Bauhaus of University, Marienstrae 15, 99423 Weimar, Germany \\ ${ }^{e}$ Department of Mechanics, University of Science, VNU HCMC, 227 Nguyen Van Cu Street, HoChiMinh City, \\ Vietnam
}

\begin{abstract}
This paper presents a new inverse tangent shear deformation theory (ITSDT) for the static, free vibration and buckling analysis of laminated composite and sandwich plates. In the present theory, shear stresses are vanished at the top and bottom surfaces of the plates and shear correction factors are no longer required. A weak form of the static, free vibration and buckling models for laminated composite and sandwich plates based on ITSDT is then derived and is numerically solved using an isogeometric analysis (IGA). The proposed formulation requires $C^{1}$-continuity generalized displacements and hence basis functions used in IGA fulfill this requirement. Numerical examples are provided to show high efficiency of the present method compared with other published solutions.
\end{abstract}

Keywords: Isogeometric analysis, laminated composite and sandwich plates, inverse trigonometric shear deformation theory

\section{Introduction}

In the past few decades, developments in science and technology have created motivations for researchers to find on new structural materials such as composite and sandwich. These materials have been used in various engineering disciplines such as aerospace engineering, automotive engineering, civil engineering, etc. Plates are an important part of many structures. Laminated composite plates are often made of several orthotropic layers and bonded together to achieve superior properties such as high stiffness and strength-to-weight ratios, long fatigue life, wear resistance, lightweight, etc. Especially, for sandwich plates, inner layers are replaced by a core which has low stiffness. Therefore, a good understanding of bending behavior, stress distribution, dynamic and buckling responses of the plates is necessary for researchers and users.

\footnotetext{
${ }^{*}$ Corresponding author. Email address: nxhung @hcmus.edu.vn (H. Nguyen-Xuan)
} 
Several laminated plate theories have been investigated for composite and sandwich plates. The classical laminate plate theory (CLPT) [1] is only suitable for thin plates. The first-order shear deformation theory (FSDT) [2], which shear deformation effect is regarded, can be applied for both moderately thick and thin plates. The FSDT does not satisfy free boundary conditions on the lower and upper surface of the plates, and hence shear correction factors need to be involved. To avoid using shear correction factors, many higher order shear deformation theories have been devised by the researchers, e.g, Ambartsumian [3], Reissner [4], Levinson [5], Reddy [6], Soldatos [7], Karama et al. [8] and Aydogdu [9], etc. Classically, first-order and higher-order theories are used the equivalent single-layer models (ESL), which consider the same degrees of freedom for all laminate layers. In addition, several other equivalent-single-layer models for laminated plates have been proposed accounting for zig-zag effects and fulfillment of interlaminar continuity. Among these the one by Mau [10], Chou and Carleone [11], Di Sciuva [12], Toledano and Murakami [13], Ren [14] and Castro et al. [15] are herein mentioned. Mixed layer-wise and equivalent-singlelayer theories based on Reissener Mixed Variational Theorem have been discussed by Carrera $[16,17,18]$. A historical review encompassing early and recent developments of advanced theories for laminated beams, plates and shells was revisited in [19]. Interested readers are addressed to that last paper for a more complete review on relevant topics.

In the effort to development of advanced computational methodologies, Hughes et al. [20] have recently proposed an isogeometric analysis (IGA) that bridges the gap between Computer Aided Design (CAD) and Finite Element Analysis (FEA). It means that the IGA uses basis functions generated from Non-Uniform Rational B-Splines (NURBS) in order to describe both the geometry and the unknown variables of the problem. Therefore, the process of meshing in IGA can be omitted and the two models for CAD and FEA integration into one. The main advantages of IGA are ability to represent exactly domains being conic sections and higher order approximation with arbitrarily high smoothness. In IGA, the exact geometry is maintained at the coarsest level of discretization and re-meshing is performed on this level without any further communication with CAD geometry. Furthermore, B-splines (or NURBS) provide a flexible way to perform refinement (or $h$-refinement), and degree elevation [21]. Isogeometric analysis has been applied to a wide range of practical mechanics problems such as structural vibrations [22], nearly incompressible linear and nonlinear problems [23], structural shape optimization [24], Kirchhoff-Love shell [25, 26, 27], isotropic Reissner-Mindlin shell [28], laminated composite/functionally graded plates based on FSDT [29, 30, 31]/HSDT [32, 33], and rotation-free shells [34], etc.

In this paper, an effectively approximate formulation based on a NURBS-based isogeometric analysis associated with a new inverse tangent shear deformation theory (ITSDT) is presented for static, free vibration and buckling analysis of laminated composite and sandwich plates. An inverse tangent function can be expressed by means of Taylor expansion, that has more general form than the classical polynomial. Generalized displacements are constructed using the NURBS basis functions that can yield higher-order continuity and fulfill easily the requirement of $C^{1}$-continuity of the HSDT models. Several numerical examples are illustrated to show high effectiveness of the present method. Obtained results are well compared with exact three-dimensional elasticity, analytical or semi-analytical and other numerical solutions.

The paper is arranged as follows: a brief on the B-spline and NURBS surface is described in 
section 2. Section 3 presents a formulation of a NURBS-based isogeometric analysis for composite sandwich plates. Several numerical examples are provided in section 4. Finally we close our paper with some concluding remarks.

\section{A brief of NURBS functions}

\subsection{Knot vectors and basis functions}

Let $\Xi=\left[\xi_{1}, \xi_{2}, \ldots, \xi_{n+p+1}\right]$ be a nondecreasing sequence of parameter values, $\xi_{i} \leq \xi_{i+1}, i=$ $1, \ldots, n+p$. The $\xi_{i}$ are called knots, and $\Xi$ is the set of coordinates in the parametric space. If all knots are equally spaced the knot vector is called uniform. If the first and the last knots are repeated $p+1$ times, the knots vector is described as open. A B-spline basis function is $C^{\infty}$ continuous inside a knot span and $C^{p-1}$ continuous at a single knot. A knot value can appear more than once and is then called a multiple knot. At a knot of multiplicity $k$ the continuity is $C^{p-k}$. Given a knot vector, the B-spline basis functions $N_{i, p}(\xi)$ of order $p=0$ are defined as follows

$$
N_{i, 0}(\xi)= \begin{cases}1 & \xi_{i} \leq \xi<\xi_{i+1} \\ 0 & \text { otherwise }\end{cases}
$$

The basis functions of order $p>0$ is defined by the following recursion formula [35]

$$
N_{i, p}(\xi)=\frac{\xi-\xi_{i}}{\xi_{i+p}-\xi_{i}} N_{i, p-1}(\xi)+\frac{\xi_{i+p+1}-\xi}{\xi_{i+p+1}-\xi_{i+1}} N_{i+1, p-1}(\xi) \quad \text { with } p=(1,2,3, \ldots)
$$

For $p=0$ and 1, the basis functions of isogeometric analysis are identical to those of standard piecewise constant and linear finite elements, respectively. In IGA, the basis functions with $p \geq 2$ are considered [20]. Fig. 1 illustrates a set of one-dimensional and two-dimensional cubic B-spline basis functions for open uniform knot vectors $\Xi=\left\{0,0,0,0, \frac{1}{2}, 1,1,1,1\right\}$.

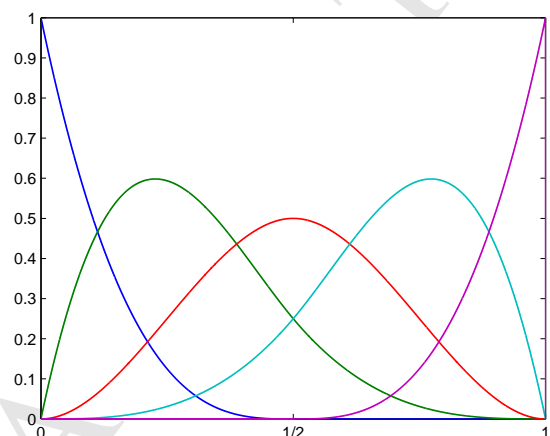

(a) $1 \mathrm{D}$

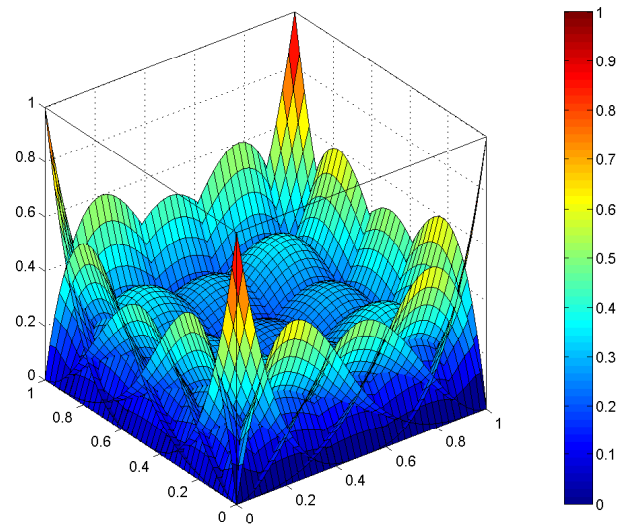

(b) $2 \mathrm{D}$

Figure 1: 1D and 2D cubic B-spline basis functions. 


\subsection{NURBS surface}

The B-spline curve is defined as

$$
\mathbf{C}(\xi)=\sum_{i=1}^{n} N_{i, p}(\xi) \mathbf{P}_{i}
$$

where $\mathbf{P}_{i}$ are the control points, $n$ denotes the number of control points and $N_{i, p}(\xi)$ is the $p^{t h}$-degree B-spline basis function defined on the open knot vector.

Given two knot vectors $\Xi=\left\{\xi_{1}, \xi_{2}, \ldots, \xi_{n+p+1}\right\}$ and $\mathscr{H}=\left\{\eta_{1}, \eta_{2}, \ldots, \eta_{m+q+1}\right\}$ and a control net $\mathbf{P}_{i, j}$, a tensor-product B-spline surface is defined as

$$
\mathscr{S}(\xi, \eta)=\sum_{i=1}^{n} \sum_{j=1}^{m} N_{i, p}(\xi) M_{j, q}(\eta) \mathbf{P}_{i, j}
$$

where $N_{i, p}(\xi)$ and $M_{j, q}(\eta)$ are the B-spline basis functions defined on the knot vectors $\Xi$ and $\mathscr{H}$, respectively.

In a finite element context, we identify the logical coordinates $(i, j)$ of the B-spline surface with the traditional notation of a "node" $I$ [28] and rewrite Eq. (4) as follows

$$
\mathscr{S}(\xi, \eta)=\sum_{I}^{n \times m} N_{I}^{b}(\xi, \eta) \mathbf{P}_{I}
$$

where $N_{I}^{b}(\xi, \eta)=N_{i, p}(\xi) M_{j, q}(\eta)$ is the shape function associated with a node $I$. The superscript $b$ indicates that $N_{I}^{b}(\xi, \eta)$ is a B-spline shape function.

Non-uniform rational B-splines (NURBS) are obtained by augmenting every point in the control mesh $\mathbf{P}_{I}$ with the weights $w_{I}^{g}$. The weighting function is constructed as follows

$$
w_{g}(\xi, \eta)=\sum_{I=1}^{n \times m} N_{I}^{b}(\xi, \eta) w_{I}^{g}
$$

The NURBS surfaces are then defined by

$$
\mathscr{S}(\xi, \eta)=\frac{\sum_{I=1}^{n \times m} N_{I}^{b}(\xi, \eta) w_{I}^{g} \mathbf{P}_{I}}{w_{g}(\xi, \eta)}=\sum_{I=1}^{n \times m} N_{I}(\xi, \eta) \mathbf{P}_{I}
$$

where $N_{I}(\xi, \eta)=N_{I}^{b}(\xi, \eta) w_{I}^{g} / w_{g}(\xi, \eta)$ are NURBS basis functions.

\section{An isogeometric laminated plate formulation using a new inverse tangent shear deforma- tion theory}

\subsection{The displacements, strains and stresses in plates}

Let $\Omega$ be the domain in $\mathbb{R}^{2}$ occupied by the mid-plane of the plate and $u_{0}, v_{0}, w$ and $\beta=$ $\left(\beta_{x}, \beta_{y}\right)^{T}$ denote the displacement components in the $x, y, z$ directions and the rotations in the $x-z$ and $y-z$ planes (or the-y and the-x axes), respectively, see Fig. 2. A generalized five-parameter 
Table 1: Several trigonometric shear deformation theories

\begin{tabular}{ll}
\hline Theory & $f(z)$ \\
\hline Arya [38] (2002) & $\sin \left(\frac{\pi z}{h}\right)$ \\
Touratier [39] (1991) & $\frac{h}{\pi} \sin \left(\frac{\pi z}{h}\right)$ \\
Soldatos [7] (1992) & $h \sinh \frac{z}{h}-\mathrm{zcosh}\left(\frac{1}{2}\right)$ \\
Proposed model & $h \arctan \left(\frac{2 z}{h}\right)-z$ \\
\hline
\end{tabular}

displacement field based on higher-order shear deformation theories [7, 9, 36, 37] are defined as follows

$$
\begin{aligned}
& u(x, y, z)=u_{0}(x, y)-z \frac{\partial w}{\partial x}+f(z) \beta_{x}(x, y) \\
& v(x, y, z)=v_{0}(x, y)-z \frac{\partial w}{\partial y}+f(z) \beta_{y}(x, y) \\
& w(x, y, z)=w(x, y)
\end{aligned}
$$

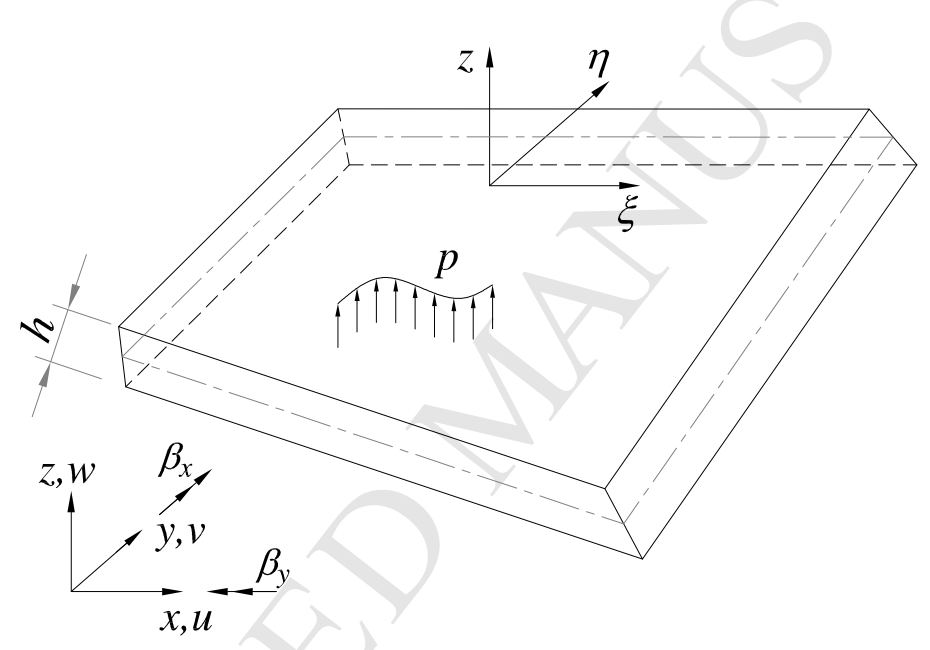

Figure 2: Geometry of a plate.

where $f(z)$ is shape function determining the distribution of the transverse shear strains and stresses through the thickness of plates. This distribution function is chosen so that tangential stress-free boundary conditions at the top and bottom surfaces of the plates are satisfied. In the present formulation, an inverse tangent function is proposed. Several trigonometric shape functions derived by other researchers are listed in Table 1. Shape functions and derivation of its through the thickness of the plate are illustrated in Fig. 3. It be can seen that the zeros shear stress conditions at the top and bottom surfaces of the plates are obtained.

The in-plane strain vector $\varepsilon_{p}=\left[\begin{array}{lll}\varepsilon_{x x} & \varepsilon_{y y} & \gamma_{x y}\end{array}\right]^{T}$ can be rewritten as

$$
\varepsilon_{p}=\varepsilon_{0}+z \varepsilon_{1}+f(z) \varepsilon_{2}
$$

where 

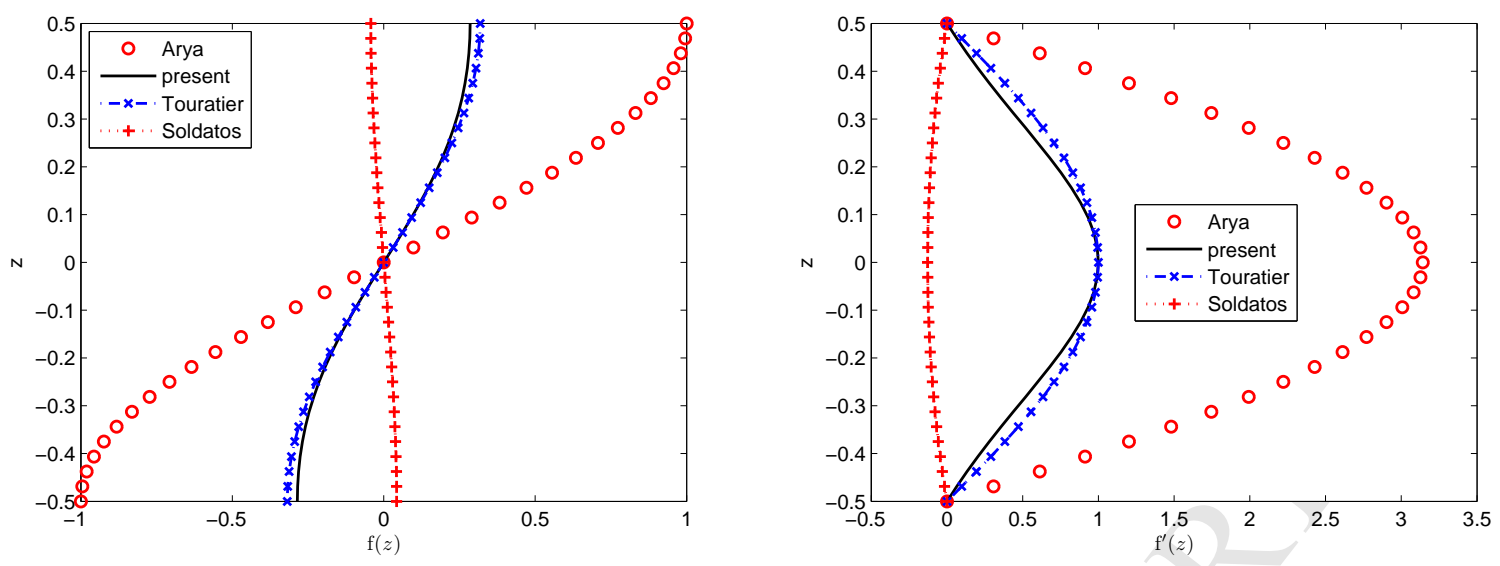

Figure 3: Shape functions $f(z))$ and its derivation through the thickness of the plate.

$$
\varepsilon_{0}=\left[\begin{array}{c}
\frac{\partial u_{0}}{\partial x} \\
\frac{\partial v_{0}}{\partial y} \\
\frac{\partial u_{0}}{\partial y}+\frac{\partial v_{0}}{\partial x}
\end{array}\right] ; \quad \varepsilon_{1}=\left[\begin{array}{c}
-\frac{\partial^{2} w}{\partial x^{2}} \\
-\frac{\partial^{2} w}{\partial y^{2}} \\
-2 \frac{\partial^{2} w}{\partial x \partial y}
\end{array}\right] ; \quad \varepsilon_{2}=\left[\begin{array}{c}
\frac{\partial \beta_{x}}{\partial x} \\
\frac{\partial \beta_{y}}{\partial y} \\
\frac{\partial \beta_{x}}{\partial y}+\frac{\partial \beta_{y}}{\partial x}
\end{array}\right]
$$

and the transverse shear strain vector $\gamma=\left[\begin{array}{lll}\gamma_{x z} & \gamma_{y z}\end{array}\right]^{T}$ has the following form

$$
\left[\gamma_{x z} \gamma_{y z}\right]^{T}=g(z) \varepsilon_{s}
$$

where

$$
\varepsilon_{s}=\left[\begin{array}{l}
\beta_{x} \\
\beta_{y}
\end{array}\right] ; \quad g(z)=\frac{d f(z)}{d z}
$$

Neglecting $\sigma_{z}$ for each orthotropic layer, the constitutive equation of an orthotropic layer in the local coordinate system is derived from Hookes law for a plane stress by

$$
\left\{\begin{array}{l}
\sigma_{1}^{(k)} \\
\sigma_{2}^{(k)} \\
\tau_{12}^{(k)} \\
\tau_{13}^{(k)} \\
\tau_{23}^{(k)}
\end{array}\right\}=\left[\begin{array}{ccccc}
Q_{11} & Q_{12} & 0 & 0 & 0 \\
Q_{12} & Q_{22} & 0 & 0 & 0 \\
0 & 0 & Q_{33} & 0 & 0 \\
0 & 0 & 0 & Q_{55} & 0 \\
0 & 0 & 0 & 0 & Q_{44}
\end{array}\right]^{(k)}\left\{\begin{array}{c}
\varepsilon_{1}^{(k)} \\
\varepsilon_{2}^{(k)} \\
\gamma_{12}^{(k)} \\
\gamma_{13}^{(k)} \\
\gamma_{23}^{(k)}
\end{array}\right\}
$$

where subscripts 1 and 2 are the directions of the fiber and in-plane normal to fiber, respectively, subscript 3 indicates the direction normal to the plate; and the reduced stiffness components, $Q_{i j}^{(k)}$, 
are given by

$$
\begin{aligned}
& Q_{11}^{(k)}=\frac{E_{1}^{(k)}}{1-v_{12}^{(k)} v_{21}^{(k)}}, Q_{12}^{(k)}=\frac{v_{12}^{(k)} E_{2}^{(k)}}{1-v_{12}^{(k)} v_{21}^{(k)}}, Q_{22}^{(k)}=\frac{E_{2}^{(k)}}{1-v_{12}^{(k)} v_{21}^{(k)}} \\
& Q_{33}^{(k)}=G_{12}^{(k)}, Q_{55}^{(k)}=G_{13}^{(k)}, Q_{44}^{(k)}=G_{23}^{(k)}
\end{aligned}
$$

in which $E_{1}^{(k)}, E_{2}^{(k)}, G_{12}^{(k)}, G_{23}^{(k)}, v_{12}^{(k)}$ and $v_{21}^{(k)}$ are independent material properties for each layer.

The laminate is usually made of several orthotropic layers. Each layer must be transformed into the laminate coordinate system $(x, y, z)$. The stress - strain relationship is given as

$$
\left\{\begin{array}{l}
\sigma_{x x} \\
\sigma_{y y} \\
\tau_{x y} \\
\tau_{x z} \\
\tau_{y z}
\end{array}\right\}^{(k)}=\left[\begin{array}{ccccc}
\bar{Q}_{11} & \bar{Q}_{12} & \bar{Q}_{16} & 0 & 0 \\
\bar{Q}_{21} & \bar{Q}_{22} & \bar{Q}_{26} & 0 & 0 \\
\bar{Q}_{61} & \bar{Q}_{62} & \bar{Q}_{66} & 0 & 0 \\
0 & 0 & 0 & \bar{Q}_{55} & \bar{Q}_{54} \\
0 & 0 & 0 & \bar{Q}_{45} & \bar{Q}_{44}
\end{array}\right]^{(k)}\left\{\begin{array}{c}
\varepsilon_{x x} \\
\varepsilon_{y y} \\
\gamma_{x y} \\
\gamma_{x z} \\
\gamma_{y z}
\end{array}\right\}
$$

where $\bar{Q}_{i j}$ is the transformed material constant matrix (see [40] for more details).

\subsection{Weak form}

A weak form of the static model for composite sandwich plates using HSDT can be briefly expressed as

$$
\int_{\Omega} \delta \varepsilon_{p}^{T} \overline{\mathbf{D}} \varepsilon_{p} \mathrm{~d} \Omega+\int_{\Omega} \delta \varepsilon_{s}^{T} \mathbf{D}^{s} \varepsilon_{s} \mathrm{~d} \Omega=\int_{\Omega} \delta w \bar{p} \mathrm{~d} \Omega
$$

where $\bar{p}, \varepsilon_{p}=\left[\begin{array}{lll}\varepsilon_{0} & \varepsilon_{1} & \varepsilon_{2}\end{array}\right]^{T}$ and $\varepsilon_{s}$ are the transverse loading per unit area, in-plane strains and transverse shear strains vector, respectively, and

$$
\begin{gathered}
\overline{\mathbf{D}}=\left[\begin{array}{ccc}
\mathbf{A} & \mathbf{B} & \mathbf{E} \\
\mathbf{B} & \mathbf{D} & \mathbf{F} \\
\mathbf{E} & \mathbf{F} & \mathbf{H}
\end{array}\right] \\
\left(A_{i j}, B_{i j}, D_{i j}, E_{i j}, F_{i j}, H_{i j}\right)=\int_{-h / 2}^{h / 2}\left(1, z, z^{2}, f(z), z f(z), f^{2}(z)\right) \bar{Q}_{i j} \mathrm{~d} z \quad(i, j=1,2,6) \\
D_{i j}^{s}=\int_{-h / 2}^{h / 2}\left(g^{2}(z)\right) \bar{Q}_{i j} \mathrm{~d} z \quad(i, j=4,5)
\end{gathered}
$$

For the free vibration analysis of composite sandwich plates using HSDT, a weak form may be derived from the dynamic form of the principle of virtual work

$$
\int_{\Omega} \delta \varepsilon_{p}^{T} \overline{\mathbf{D}} \varepsilon_{p} \mathrm{~d} \Omega+\int_{\Omega} \delta \varepsilon_{s}^{T} \mathbf{D}^{s} \varepsilon_{s} \mathrm{~d} \Omega=\int_{\Omega} \delta \tilde{\mathbf{u}}^{T} \mathbf{m} \ddot{\tilde{\mathbf{u}}} \mathrm{d} \Omega
$$

where

$$
\mathbf{m}=\left[\begin{array}{ccc}
I_{1} & I_{2} & I_{4} \\
I_{2} & I_{3} & I_{5} \\
I_{4} & I_{5} & I_{6}
\end{array}\right] ; \quad\left(I_{1}, I_{2}, I_{3}, I_{4}, I_{5}, I_{6}\right)=\int_{-h / 2}^{h / 2} \rho\left(1, z, z^{2}, f(z), z f(z), f^{2}(z)\right) \mathrm{d} z
$$

in which $\tilde{\mathbf{u}}=\left[\begin{array}{lll}\mathbf{u}_{0} & \mathbf{u}_{1} & \mathbf{u}_{2}\end{array}\right]^{T}$ and 


$$
\mathbf{u}_{0}=\left[\begin{array}{c}
u_{0} \\
v_{0} \\
w
\end{array}\right] ; \quad \mathbf{u}_{1}=\left[\begin{array}{c}
-\frac{\partial w}{\partial x} \\
-\frac{\partial w}{\partial y} \\
0
\end{array}\right] ; \quad \mathbf{u}_{2}=\left[\begin{array}{c}
\beta_{x} \\
\beta_{y} \\
0
\end{array}\right]
$$

In the case of in-plane buckling analysis and assuming pre-buckling stresses $\hat{\sigma}_{0}$, nonlinear strains are taken into account in the weak form as

$$
\int_{\Omega} \delta \varepsilon_{p}^{T} \overline{\mathbf{D}} \varepsilon_{p} \mathrm{~d} \Omega+\int_{\Omega} \delta \varepsilon_{s}^{T} \mathbf{D}^{s} \varepsilon_{s} \mathrm{~d} \Omega+h \int_{\Omega} \nabla^{T} \delta w \hat{\sigma}_{0} \nabla w \mathrm{~d} \Omega=0
$$

where $\nabla^{T}=\left[\begin{array}{ll}\partial / \partial x & \partial / \partial y\end{array}\right]$ and $\hat{\sigma}_{0}=\left[\begin{array}{cc}\sigma_{x}^{0} & \tau_{x y}^{0} \\ \tau_{x y}^{0} & \sigma_{y}^{0}\end{array}\right]$ are the gradient operator and in-plane prebuckling stresses, respectively.

Using NURBS basis functions, the field variables are the in-plane extensions, transverse deflection and the rotations at all control points, which can be expressed as

$$
\mathbf{u}=\left\{\begin{array}{l}
u_{0} \\
v_{0} \\
w \\
\beta_{x} \\
\beta_{y}
\end{array}\right\}=\sum_{I=1}^{n \times m}\left[\begin{array}{ccccc}
N_{I} & 0 & 0 & 0 & 0 \\
0 & N_{I} & 0 & 0 & 0 \\
0 & 0 & N_{I} & 0 & 0 \\
0 & 0 & 0 & N_{I} & 0 \\
0 & 0 & 0 & 0 & N_{I}
\end{array}\right]\left\{\begin{array}{c}
u_{I} \\
v_{I} \\
w_{I} \\
\beta_{x I} \\
\beta_{y I}
\end{array}\right\}=\sum_{I=1}^{n \times m} \mathbf{N}_{I} \mathbf{q}_{I}
$$

where $n \times m$ is the number basis functions, $\mathbf{N}_{I}$ and $\mathbf{q}_{I}=\left[\begin{array}{lllll}u_{I} & v_{I} & w_{I} & \beta_{x I} & \beta_{y I}\end{array}\right]^{T}$ are rational basis functions and the degrees of freedom of $\mathbf{u}$ associated with a control point $I$, respectively.

The in-plane strains, shear strains and geometrical strains are written as:

$$
\left[\begin{array}{ll}
\varepsilon_{p} & \gamma
\end{array}\right]^{T}=\sum_{I=1}^{n \times m}\left[\begin{array}{llll}
\mathbf{B}_{I}^{b 0} & \mathbf{B}_{I}^{b 1} & \mathbf{B}_{I}^{b 2} & \mathbf{B}_{I}^{s}
\end{array}\right]^{T} \mathbf{q}_{I}=\sum_{I=1}^{n \times m} \mathbf{B}_{I} \mathbf{q}_{I} ; \quad \varepsilon_{g}=\sum_{I=1}^{n \times m} \mathbf{B}_{I}^{g} \mathbf{q}_{I}
$$

where

$\mathbf{B}_{I}^{b 0}=\left[\begin{array}{ccccc}N_{I, x} & 0 & 0 & 0 & 0 \\ 0 & N_{I, y} & 0 & 0 & 0 \\ N_{I, y} & N_{I, x} & 0 & 0 & 0\end{array}\right] ; \mathbf{B}_{I}^{b 1}=\left[\begin{array}{ccccc}0 & 0 & -N_{I, x x} & 0 & 0 \\ 0 & 0 & -N_{I, y y} & 0 & 0 \\ 0 & 0 & -2 N_{I, x y} & 0 & 0\end{array}\right] ; \mathbf{B}_{I}^{b 2}=\left[\begin{array}{ccccc}0 & 0 & 0 & N_{I, x} & 0 \\ 0 & 0 & 0 & 0 & N_{I, y} \\ 0 & 0 & 0 & N_{I, y} & N_{I, x}\end{array}\right]$ and

$$
\mathbf{B}_{I}^{s}=\left[\begin{array}{ccccc}
0 & 0 & 0 & N_{I} & 0 \\
0 & 0 & 0 & 0 & N_{I}
\end{array}\right] ; \mathbf{B}_{I}=\left[\begin{array}{llll}
\mathbf{B}_{I}^{b 0} & \mathbf{B}_{I}^{b 1} & \mathbf{B}_{I}^{b 2} & \mathbf{B}_{I}^{s}
\end{array}\right]^{T}
$$

For static analysis, the stiffness formulation is written as

$$
\mathbf{K q}=\mathbf{f},
$$

For free vibration analysis, one forms

$$
\left(\mathbf{K}-\omega^{2} \mathbf{M}\right) \mathbf{q}=0
$$


And for buckling analysis, we have

$$
\left(\mathbf{K}-\lambda_{c r} \mathbf{K}_{g}\right) \mathbf{q}=0
$$

where $\mathbf{K}$ is the global stiffness matrix

$$
\begin{gathered}
\mathbf{K}=\int_{\Omega}\left\{\left[\begin{array}{l}
\mathbf{B}^{b 0} \\
\mathbf{B}^{b 1} \\
\mathbf{B}^{b 2}
\end{array}\right]^{T}\left[\begin{array}{lll}
\mathbf{A} & \mathbf{B} & \mathbf{E} \\
\mathbf{B} & \mathbf{D} & \mathbf{F} \\
\mathbf{E} & \mathbf{F} & \mathbf{H}
\end{array}\right]\left[\begin{array}{l}
\mathbf{B}^{b 0} \\
\mathbf{B}^{b 1} \\
\mathbf{B}^{b 2}
\end{array}\right]+\left(\mathbf{B}^{s}\right)^{T} \mathbf{D}^{\mathbf{s}} \mathbf{B}^{s}\right\} \mathrm{d} \Omega \\
\mathbf{f}=\int_{\Omega} \bar{p} \mathbf{N d} \Omega
\end{gathered}
$$

in which $\mathbf{f}$ is the load vectors and $\mathbf{M}$ is the global mass matrix

$$
\mathbf{M}=\int_{\Omega}\left\{\left[\begin{array}{l}
\mathbf{N}_{0} \\
\mathbf{N}_{1} \\
\mathbf{N}_{2}
\end{array}\right]^{T}\left[\begin{array}{lll}
I_{1} & I_{2} & I_{4} \\
I_{2} & I_{3} & I_{5} \\
I_{4} & I_{5} & I_{6}
\end{array}\right]\left[\begin{array}{l}
\mathbf{N}_{0} \\
\mathbf{N}_{1} \\
\mathbf{N}_{2}
\end{array}\right]\right\} \mathrm{d} \Omega
$$

where

$$
\mathbf{N}_{0}=\left[\begin{array}{ccccc}
N_{I} & 0 & 0 & 0 & 0 \\
0 & N_{I} & 0 & 0 & 0 \\
0 & 0 & N_{I} & 0 & 0
\end{array}\right] ; \mathbf{N}_{1}=\left[\begin{array}{ccccc}
0 & 0 & -N_{I, x} & 0 & 0 \\
0 & 0 & -N_{I, y} & 0 & 0 \\
0 & 0 & 0 & 0 & 0
\end{array}\right] ; \mathbf{N}_{2}=\left[\begin{array}{ccccc}
0 & 0 & 0 & N_{I} & 0 \\
0 & 0 & 0 & 0 & N_{I} \\
0 & 0 & 0 & 0 & 0
\end{array}\right]
$$

and the global geometrical stiffness matrix $\mathbf{K}_{g}$ is

$$
\mathbf{K}_{g}=\int_{\Omega}\left(\mathbf{B}^{g}\right)^{T} \tau \mathbf{B}^{g} \mathrm{~d} \Omega ; \quad \tau=h \hat{\sigma}_{0}
$$

in which $\rho, h, \omega$ and $\lambda_{c r}$ are the mass density, the thickness, the natural frequency and the critical buckling load, respectively.

\subsection{Essential boundary conditions}

In this part, we show how to impose essential boundary conditions of the isogeometric approach. For the sake of simplicity we consider several following Dirichlet boundary conditions (BCs):

- Simply supported rectangular plates:

$$
u_{0}\left(\mathbf{x}_{D}\right)=v_{0}\left(\mathbf{x}_{D}\right)=w\left(\mathbf{x}_{D}\right)=\beta_{n}\left(\mathbf{x}_{D}\right)=0
$$

where $\beta_{n}\left(\mathbf{x}_{D}\right)$ is the normal rotation constraint and $\mathbf{x}_{D}$ are control points that define the essential boundary.

- Simply supported plates with curved boundaries:

$$
u_{0}\left(\mathbf{x}_{D}\right)=v_{0}\left(\mathbf{x}_{D}\right)=w\left(\mathbf{x}_{D}\right)=0
$$


- Clamped plate model:

$$
u_{0}\left(\mathbf{x}_{D}\right)=v_{0}\left(\mathbf{x}_{D}\right)=w\left(\mathbf{x}_{D}\right)=\beta_{x}\left(\mathbf{x}_{D}\right)=\beta_{y}\left(\mathbf{x}_{D}\right)=w_{, n}\left(\mathbf{x}_{D}\right)=0
$$

It is worth noting that the enforcement of Dirichlet BCs on $u_{0}, v_{0}, w, \beta_{x}$ and $\beta_{y}$ is treated as in the standard FEM. This procedure involves only control points that define the essential boundary. However, for the derivatives $w_{, x}, w_{, y}$ occurred in (8), the enforcement of Dirichlet BCs can be solved in a special way based on stream function formulation proposed by Auricchio et al. [41]. To end this, the derivatives $w_{, x}, w_{, y}$ can be included in a compact form of the normal slope at the boundary as follows

$$
\frac{\partial w}{\partial n}=\lim _{\Delta n \rightarrow 0} \frac{w\left(n\left(\mathbf{x}_{D}\right)+\Delta n\right)-w\left(n\left(\mathbf{x}_{D}\right)\right)}{\Delta n}=0
$$

Due to $w\left(n\left(\mathbf{x}_{D}\right)\right)=0$ at $\mathbf{x}_{D}$, Eq.(29) implies that in the framework of IGA we impose simply same boundary values, i.e, zero values, on the deflection variable at control points $\mathbf{x}_{A}$ adjacent to the boundary control points $\mathbf{x}_{D}$ [41]. It be can observed that essential boundary condition using this way is very simple to implement in the isogeometric approach in comparison with other numerical methods. We will show in the next section that this procedure results in a high accuracy for analysis of multilayered plates.

\section{Numerical results and discussion}

Several examples of the laminated composite and sandwich plates for static, free vibration and buckling analysis have been presented to demonstrate the performance of the proposed theory. For the sake of simplicity and without loss of generality, we only consider the IGA with using NURBS cubic basis functions $(p=3)$. Additionally, besides the proposed model, the IGA is also applied for the first time to three different trigonometric shear deformation theory models $[38,39,7]$. The results obtained from the present solutions have been compared with other published ones. The material parameters used in this study are listed below:

- Material $I$ :

$E_{1}=25 E_{2}, G_{12}=G_{13}=0.5 E_{2}, G_{23}=0.2 E_{2}, v_{12}=0.25, \rho=1$.

- Material $I I[42]$ :

Face sheets:

$E_{1}=172.375 \mathrm{GPa}, E_{2}=E_{3}=6.895 \mathrm{GPa}, G_{12}=G_{13}=3.448 \mathrm{GPa}, G_{23}=1.379 \mathrm{GPa}$, $v_{12}=v_{23}=v_{13}=0.25$.

Core:

$E_{1}=0.2758 \mathrm{GPa}, E_{2}=0.2758 \mathrm{GPa}, E_{3}=3.4475 \mathrm{GPa}, G_{12}=G_{13}=G_{23}=0.4137 \mathrm{GPa}$, $v_{12}=0.25, v_{23}=v_{13}=0.02$.

- Material III: [43] $E_{1}=40 E_{2}, G_{12}=G_{13}=0.6 E_{2}, G_{23}=0.5 E_{2}, v_{12}=0.25, \rho=1$. 
- Material $I V$ [44]:

Face sheets:

$E_{1}=131 \mathrm{GPa}, E_{2}=E_{3}=10.34 \mathrm{GPa}, G_{12}=G_{23}=6.895 \mathrm{GPa}, G_{13}=6.205 \mathrm{GPa}, v_{12}=$ $v_{13}=0.25 ; v_{23}=0.49, \rho=1627 \mathrm{~kg} / \mathrm{m}^{3}$.

Core:

$E_{1}=6.89 \mathrm{MPa}, E_{2}=6.89 \mathrm{MPa}, E_{3}=6.89 \mathrm{MPa}, G_{12}=G_{13}=G_{23}=3.45 \mathrm{MPa}, v_{12}=v_{23}=$ $v_{13}=0, \rho=97 \mathrm{~kg} / \mathrm{m}^{3}$.

- Material $V:$ [45]

$E_{1}=2.45 E_{2}, G_{12}=G_{13}=0.48 E_{2}, G_{23}=0.2 E_{2}, v_{12}=0.23, \rho=1$.

- Material VI [46]:

Face sheets:

$E_{1} / E_{2}=19, G_{12} / E_{2}=G_{13} / E_{2}=0.52, G_{23} / E_{2}=0.338, v_{12}=v_{13}=0.32, v_{23}=0.49$.

Core:

$E_{1} / E_{2}^{f}=3.2 \times 10^{-5}, E_{2} / E_{2}^{f}=2.9 \times 10^{-5}, E_{3} / E_{2}^{f}=0.4, G_{12} / E_{2}^{f}=2.4 \times 10^{-3}$.

$G_{13} / E_{2}^{f}=7.9 \times 10^{-2}, G_{23} / E_{2}^{f}=6.6 \times 10^{-2}, v_{12}=0.99, v_{23}=v_{13}=3 \times 10^{-5}$.

in which $E_{2}^{f}$ refers to that of face sheets.

\subsection{Static analysis}

\subsubsection{Four layer $\left[0^{0} / 90^{0} / 90^{0} / 0^{0}\right]$ square laminated plate under sinusoidally distributed load}

We now consider a simply supported square laminated plate subjected to a sinusoidal load $q$, see Fig. 4. The length to width ratios is $a / b=1$ and the length to thickness ratios are $a / h=4,10$, 20, 100. Material $I$ is used. The normalized displacement and stresses are defined as

$$
\begin{gathered}
\varpi=\left(100 E_{2} h^{3}\right) w\left(\frac{a}{2}, \frac{a}{2}, 0\right) /\left(q a^{4}\right) ; \bar{\sigma}_{x x}=\frac{h^{2}}{q b^{2}} \sigma_{x x}(a / 2, a / 2, h / 2) \\
\bar{\sigma}_{y y}=\frac{h^{2}}{q b^{2}} \sigma_{y y}(a / 2, a / 2, h / 4) ; \bar{\tau}_{x y}=\frac{h^{2}}{q b^{2}} \sigma_{x y}(0,0, h / 2) \\
\bar{\tau}_{x z}=\frac{h}{q b} \sigma_{x z}(0, b / 2,0) ; \bar{\tau}_{y z}=\frac{h}{q b} \sigma_{y z}(a / 2,0,0)
\end{gathered}
$$

We first investigate the convergence of the normalization displacement and stresses at $a / h=4$. The plate is modeled with $9 \times 9,13 \times 13$ and $17 \times 17$ elements as shown in Fig. 5. The exact 3D elasticity solution of this problem was given by Pagano [47]. Table 7 shows the convergence of the normalization displacement and stresses of the IGA based on the present theory and the different trigonometric shear deformation theories (dTrSDTs) $[38,39,7]$. The relative error percentages compared with the exact 3D elasticity solution [47] are given in a parentheses. It be can observed that the obtained results agree very well with the exact values. Compared to the different trigonometric shear deformation theories, the IGA using the present theory produces more accurate results for both displacement and stresses.

For a comparison, the normalized displacement and stresses of a four layer simply supported square plate are computed using $17 \times 17 \mathrm{~B}$-spline elements. The obtained results of the IGA based on the present theory are compared with those of the several other methods based on other higher-order shear deformation theories such as the closed form solution (CSF) based on the HSDT by Reddy 
[6], the finite strip method (FSM) based on HSDT by Akhras et al.[48], the multiquadric radial basis function method (RBFs) based on a finite point formulation and HSDT by Ferreira et al. [49], the closed form solution based on a trigonometric shear deformation theory (TrSDT) by Mantari et al. [50], the closed form solution based on an exponential shear deformation theory (ESDT) by Karama et al. [51], the IGA based on the dTrSDTs [38, 39, 7] and an exact 3D elasticity approach studied by Pagano [47]. Table 3 is provided the comparison between the present method and other methods. It is seen that the IGA based on the present theory shows a strong competitor to other reference ones for all ratios $a / h$. The normalized displacement and stresses of the present approach are in excellent agreement with the exact solution [47]. It is seen for thick plate cases $(a / h=4,10)$ that the ITSDT yields better results than the HSDT [6, 48, 49], ESDT [51] and the dTrSDTs $[38,39,7]$ in comparison with the exact solution [47]. For thin plate case $(a / h=100)$, the difference between the solutions are not significant for all displacement and stresses. Fig. 6 plots the distribution of stresses through the thickness of a four-layer the square plate with $a / h$ $=4$ and 10, respectively. It is worth noting that the present ITSDT model reflects well the shear stresses profiles through the plate thickness. From Table 3, it is worth noting that results derived from two published models in [38] and [39] are coincided. Therefore in next examples, only the model provided in [38] is illustrated.

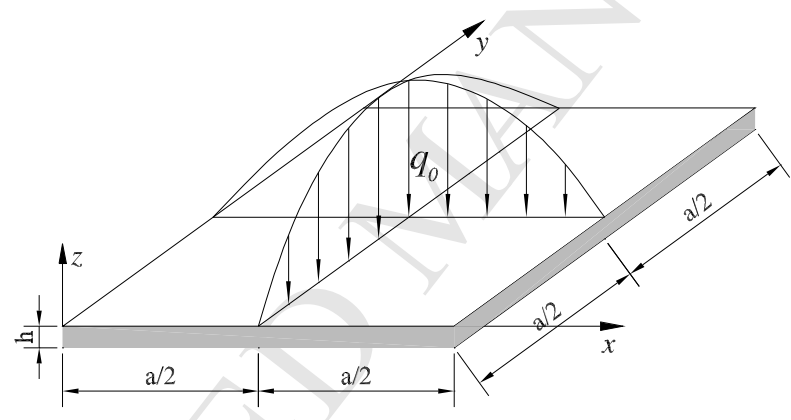

Figure 4: Geometry of a square laminated plate under sinusoidally and uniformly distributed load.

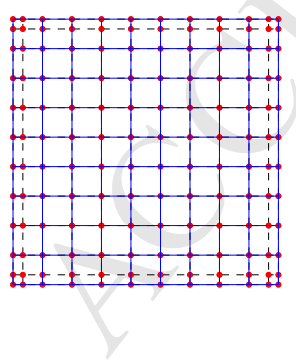

(a)

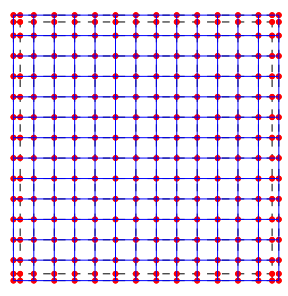

(b)

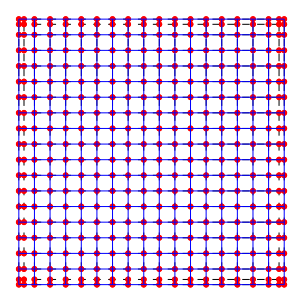

(c)

Figure 5: Meshes and control net of a square plate using cubic elements: a) $9 \times 9$; b) $13 \times 13$ and c) $17 \times 17$. 
Table 2: The convergence of the normalized displacement and stresses of a four-layer $\left[0^{0} / 90^{0} / 90^{0} / 0^{0}\right]$ laminated composite square plate $(a / h=4)$

\begin{tabular}{|c|c|c|c|c|}
\hline \multirow[t]{2}{*}{ Method } & \multirow[t]{2}{*}{ Nor. sol. } & \multicolumn{3}{|c|}{ Mesh } \\
\hline & & $9 \times 9$ & $13 \times 13$ & $17 \times 17$ \\
\hline IGA (Arya [38]) & $\bar{w}$ & $1.9086(2.32 \%)$ & $1.9087(2.32 \%)$ & $1.9088(2.31 \%)$ \\
\hline IGA (Toutatier [39]) & & $1.9086(2.32 \%)$ & $1.9087(2.32 \%)$ & $1.9088(2.31 \%)$ \\
\hline IGA (Soldatos [7]) & & $1.8919(3.18 \%)$ & $1.8920(3.17 \%)$ & $1.8920(3.17 \%)$ \\
\hline IGA (present) & & $1.9256(1.45 \%)$ & $1.9257(1.45 \%)$ & $1.9258(1.44 \%)$ \\
\hline Elasticity [47] & & - & - & 1.954 \\
\hline IGA (Arya [38]) & $\bar{\sigma}_{x x}$ & $0.6863(4.68 \%)$ & $0.6845(4.93 \%)$ & $0.6839(5.01 \%)$ \\
\hline IGA (Toutatier [39]) & & $0.6863(4.68 \%)$ & $0.6845(4.93 \%)$ & $0.6839(5.01 \%)$ \\
\hline IGA (Soldatos [7]) & & $0.6669(7.37 \%)$ & $0.665(7.64 \%)$ & $0.6644(7.72 \%)$ \\
\hline IGA (present) & & $0.7186(0.19 \%)$ & $0.7169(0.43 \%)$ & $0.7164(0.50 \%)$ \\
\hline Elasticity [47] & & - & - & 0.720 \\
\hline IGA (Arya [38]) & $\bar{\sigma}_{y y}$ & $0.6337(4.85 \%)$ & $0.6344(4.74 \%)$ & $0.6346(4.71 \%)$ \\
\hline IGA (Toutatier [39]) & & $0.6337(4.85 \%)$ & $0.6344(4.74 \%)$ & $0.6346(4.71 \%)$ \\
\hline IGA (Soldatos [7]) & & $0.6307(5.30 \%)$ & $0.6314(5.20 \%)$ & $0.6316(5.17 \%)$ \\
\hline IGA (present) & & $0.6372(4.32 \%)$ & $0.6378(4.23 \%)$ & $0.6381(4.19 \%)$ \\
\hline Elasticity [47] & & - & - & 0.666 \\
\hline IGA (Arya [38]) & $\bar{\sigma}_{x y}$ & $0.0450(3.64 \%)$ & $0.0450(3.64 \%)$ & $0.0450(3.64 \%)$ \\
\hline IGA (Toutatier [39]) & & $0.0450(3.64 \%)$ & $0.0450(3.64 \%)$ & $0.0450(3.64 \%)$ \\
\hline IGA (Soldatos [7]) & & $0.0439(6.00 \%)$ & $0.0439(6.00 \%)$ & $0.0439(6.00 \%)$ \\
\hline IGA (present) & & $0.0467(0.00 \%)$ & $0.0467(0.00 \%)$ & $0.0467(0.00 \%)$ \\
\hline Elasticity [47] & & & - & 0.0467 \\
\hline IGA (Arya [38]) & & $0.2163(19.89 \%)$ & $0.2162(19.93 \%)$ & $0.2162(19.93 \%)$ \\
\hline IGA (Toutatier [39]) & & $0.2163(19.89 \%)$ & $0.2162(19.93 \%)$ & $0.2162(19.93 \%)$ \\
\hline IGA (Soldatos [7] & & $0.2056(23.85 \%)$ & $0.2055(23.89 \%)$ & $0.2055(23.89 \%)$ \\
\hline IGA (present) & & $0.2397(11.22 \%)$ & $0.2396(11.26 \%)$ & $0.2396(11.26 \%)$ \\
\hline Elasticity [47] & & - & - & 0.270 \\
\hline IGA (Arya [38]) & $\bar{\sigma}_{y z}$ & $0.2462(15.40 \%)$ & $0.2461(15.43 \%)$ & $0.2461(15.43 \%)$ \\
\hline IGA (Toutatier [39]) & & $0.2462(15.40 \%)$ & $0.2461(15.43 \%)$ & $0.2461(15.43 \%)$ \\
\hline IGA (Soldatos [7]) & & $0.2383(18.11 \%)$ & $0.2382(18.14 \%)$ & $0.2382(18.14 \%)$ \\
\hline IGA (present) & & $0.2624(9.83 \%)$ & $0.2624(9.83 \%)$ & $0.2624(9.83 \%)$ \\
\hline Elasticity [47] & & - & - & 0.291 \\
\hline
\end{tabular}


Table 3: The normalized displacement and stresses of a supported simply $\left[0^{0} / 90^{0} / 90^{0} / 0^{0}\right]$ square laminated plate under a sinusoidally distributed load.

\begin{tabular}{|c|c|c|c|c|c|c|c|}
\hline$a / h$ & Method & $\bar{w}$ & $\bar{\sigma}_{x x}$ & $\bar{\sigma}_{y y}$ & $\bar{\sigma}_{x y}$ & $\bar{\sigma}_{x z}$ & $\bar{\sigma}_{y z}$ \\
\hline \multirow{10}{*}{7} & CFS-HSDT [6] & 1.8939 & 0.6806 & 0.6463 & 0.0450 & 0.2109 & 0.2390 \\
\hline & FSM-HSDT [48] & 8937 & 0.6651 & 0.6322 & 0.0440 & 0.2064 & - \\
\hline & BFs-HSDT [49] & 8864 & 6659 & 0.6313 & 0.0433 & 0.1352 & 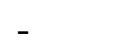 \\
\hline & FS-TrSDT [50] & 921 & .74 & 0.635 & 0.048 & 0.254 & 0.269 \\
\hline & FS-ESDT [51] & 919 & 699 & 636 & 0459 & 0.226 & 0.253 \\
\hline & lasticity [47] & 954 & 720 & 666 & 0467 & 0.270 & 0.291 \\
\hline & GA (Arya ) & 9088 & 0.6839 & 0.6346 & 0.0450 & 0.2162 & 0.2461 \\
\hline & IGA (Touratier [39]) & 1.9088 & 0.6839 & 0.6346 & 0.0450 & 0.2162 & 0.2461 \\
\hline & IGA (Soldatos ) & 1.8920 & 0.6644 & 0.6316 & 0.0439 & 0.2055 & 0.2382 \\
\hline & IGA (present) & 1.9258 & 0.7164 & 0.6381 & 0.0467 & 0.2396 & 0.2624 \\
\hline \multirow[t]{10}{*}{10} & CFS-HSDT [6] & 0.7149 & & 0 . & 0.0273 & 97 & 0.1 \\
\hline & SM & 7147 & 56 & 0.3888 & 0.0268 & 0.2640 & - \\
\hline & $\mathrm{BFs}$ & 7153 & 466 & 0.4383 & 0267 & 0.3347 & - \\
\hline & CFS & 30 & 51 & 0.3 & 028 & 0.335 & 0.176 \\
\hline & CFS- & 724 & 553 & 0.393 & 0.027 & .294 & 163 \\
\hline & astic & 743 & 559 & 0.403 & 0.0276 & 0.301 & 0.196 \\
\hline & IGA (Arya ) & 7198 & 0.5486 & 0.3905 & 0.0270 & 0.2787 & 0.1582 \\
\hline & IGA $(1$ & 0.7198 & 0.5486 & 0.3905 & 0.0270 & 0.2787 & 0.1582 \\
\hline & IGA $(s$ & 0.7142 & 0.5449 & 0.3881 & 0.0267 & 0.2627 & 0.1526 \\
\hline & IGA & 7272 & 0.5552 & 0.3937 & 0.0273 & 0.3133 & 0.1704 \\
\hline \multirow[t]{10}{*}{20} & T [6] & 61 & 0.5 & & 33 & 0.2883 & 0.1230 \\
\hline & & 0 & & & & 25 & - \\
\hline & $\mathrm{R}$ & 70 & 55 & 0. & 0.0228 & 0.3818 & - \\
\hline & CFS & 10 & 0.542 & 0.306 & 0.023 & 0.323 & 0.132 \\
\hline & CFS & 509 & 0.541 & 0.306 & 0.023 & 0.316 & 0.131 \\
\hline & Elasticity [47] & 51 & 0.5 & 0.3 & 0.0230 & 0.328 & 0.156 \\
\hline & IGA (Ary: & 0.5075 & 0.5395 & 0.3046 & 0.0228 & 0.2989 & 0.1272 \\
\hline & IGA (T & 0.50 & 0.5395 & 0.3046 & .0228 & 0.2989 & 0.1272 \\
\hline & IGA $(s$ & 0.5059 & 0.5385 & 0.3038 & 0.0228 & 0.2810 & 0.1231 \\
\hline & IGA (present) & 0.5098 & 0 & 0. & 29 & 72 & 0 \\
\hline \multirow[t]{10}{*}{100} & CFS-HSDT [ & & & & & & 0.1120 \\
\hline & & & & & & & - \\
\hline & & & & & 215 & & - \\
\hline & & & & 0.271 & & & 0.119 \\
\hline & & & & 0.2 & 0. & 0.324 & 0.118 \\
\hline & Elasti & 4347 & & 0.2 & 0.0214 & 0.339 & 0.141 \\
\hline & IGA & 4344 & 0. & 0.2705 & 0.0213 & 0.3069 & 0.1148 \\
\hline & IGA & 4344 & 0 & 0.2705 & 0.0213 & 0.3069 & 0.1148 \\
\hline & IGA & 12 & 0.5379 & 0.2704 & 0.0213 & 0.2882 & 0.1114 \\
\hline & $\mathrm{IG} t$ & & $\begin{array}{l}0.5380 \\
14\end{array}$ & 0.2705 & 0.0213 & 0.3467 & 22 \\
\hline
\end{tabular}



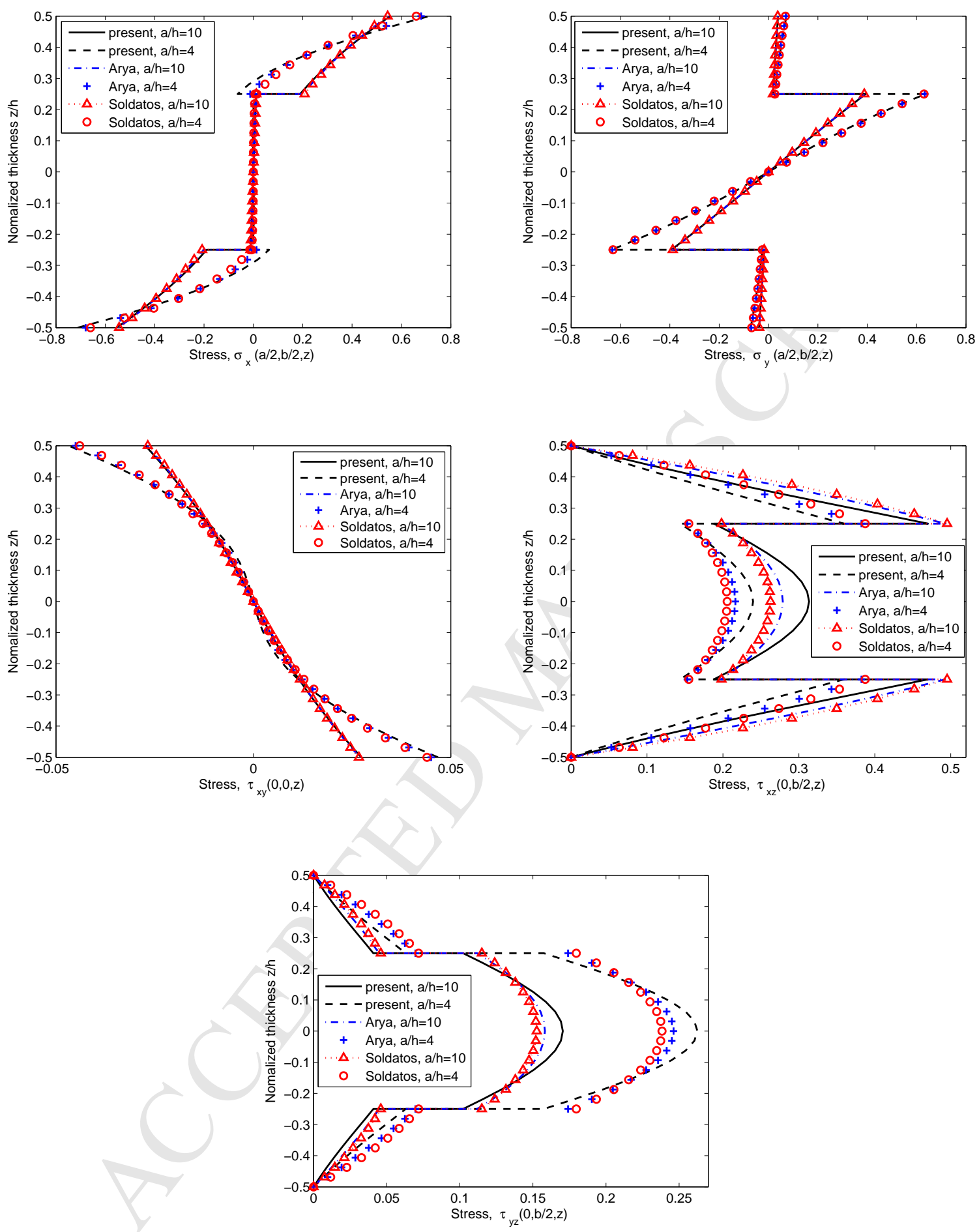

Figure 6: The distribution of stresses through the thickness of a four-layer $\left[0^{0} / 90^{0} / 90^{0} / 0^{0}\right]$ square plate under a sinusoidally distributed load. 


\subsubsection{Three layer $\left[0^{0} / 90^{0} / 0^{0}\right]$ square laminated plate under sinusoidally distributed load}

A three-layer $\left[0^{0} / 90^{0} / 0^{0}\right]$ simply supported square laminated plate subjected to a sinusoidal load $q$ as previous example is considered. The length to thickness ratios are $a / h=4,10,20,100$, respectively. Material $I$ is also used. The normalized displacement and stresses of a square plate are defined as:

$$
\begin{gathered}
\varpi=\left(100 E_{2} h^{3}\right) w\left(\frac{a}{2}, \frac{a}{2}, 0\right) /\left(q a^{4}\right) ; \bar{\sigma}_{x x}=\frac{h^{2}}{q b^{2}} \sigma_{x x}(a / 2, a / 2, h / 2) \\
\bar{\sigma}_{y y}=\frac{h^{2}}{q b^{2}} \sigma_{y y}(a / 2, a / 2, h / 6) ; \bar{\tau}_{x y}=\frac{h^{2}}{q b^{2}} \sigma_{x y}(0,0, h / 2) \\
\bar{\tau}_{x z}=\frac{h}{q b} \sigma_{x z}(0, b / 2,0) ; \bar{\tau}_{y z}=\frac{h}{q b} \sigma_{y z}(a / 2,0,0)
\end{gathered}
$$

Table 4 lists the results of the IGA based on present theory using $17 \times 17$ cubic B-Spline elements. Numerical solutions are compared to those reported in the literature such as the closed form solution based on HSDT by Reddy [6], the closed form solution based on ESDT by Karama et al. [51], the closed form solution based on TrSDT by Mantari et al. [50], the closed form solution based on inverse hyperbolic shear deformation theory (IHSDT) by Grover et al. [52], the IGA solution based on the dTrSDTs [38, 7] and an exact elasticity solution by Pagano [47]. The obtained results indicate a reasonably good agreement with other available ones for all $a / h$ ratios. The normalized displacement and stresses derived from the present theory are more accuracy than those of HSDT [6] and ESDT [51]. Also, the stresses profiles through the thickness of the three-layer square laminated plate with $a / h=4$ and 10, respectively, are again displayed in Fig. 7.

\subsubsection{Sandwich $\left(0^{0} /\right.$ core $\left./ 0^{0}\right)$ square plate subjected under sinusoidally distributed load}

Let us consider a sandwich $\left(0^{0} /\right.$ core $\left./ 0^{0}\right)$ simply supported square plate subjected to a sinusoidally distributed load. The thickness of each face sheet is fixed at $h / 10$. The length to thickness ratios are used as $a / h=4,10,20,50,100$. Material $I I$ is used in this example. The plate is modeled by $17 \times 17 \mathrm{~B}$-spline elements. The normalized transverse displacement and stresses are defined as follows

$$
\begin{gathered}
\bar{w}=100 h^{3} E_{2} w\left(\frac{a}{2}, \frac{a}{2}, \frac{h}{2}\right) / q_{0} a^{4}, \quad \bar{\sigma}_{x}=h^{2} \sigma_{x}\left(\frac{a}{2}, \frac{a}{2}, \frac{h}{2}\right) / q_{0} a^{2}, \\
\bar{\sigma}_{y}=h^{2} \sigma_{y}\left(\frac{a}{2}, \frac{a}{2}, \frac{h}{2}\right) / q_{0} a^{2}, \quad \bar{\sigma}_{x y}=h^{2} \sigma_{x y}\left(0,0, \frac{h}{2}\right) / q_{0} a^{2}, \\
\bar{\sigma}_{x z}=h \sigma_{x z}\left(0, \frac{b}{2}, 0\right) / a, \quad \bar{\sigma}_{y z}=h \sigma_{y z}\left(\frac{a}{2}, 0,0\right) / a .
\end{gathered}
$$

Table 5 summarizes normalized transverse displacement and stresses derived from the IGA based on present theory in comparison with the exact elasticity solution by Pagano [47], the closed form solution by Kant and Swaminathan [42] based on FSDT \& HSDT and our isogeometric approach based on the dTrSDTs [38, 7]. Again, the present results are in good agreement with the exact elasticity one [47] and also the analytical one [42]. It is evident that FSDT model leads to inaccurate results compared to other models when the plate becomes thicker. Henceforth, this shortcoming motivates the development of higher order shear deformation theories. It is worth noting that our proposed method is completely relied on the numerical approximation and can provide strongly competitive solutions to well-known analytical approaches. Fig. 8 exhibits the distribution of stresses through the thickness of the plate using the present theory and the dTrSDTs 
Table 4: The normalized displacement and stresses of a supported simply $\left[0^{0} / 90^{\circ} / 0^{0}\right]$ square laminated plate under a sinusoidally distributed load

\begin{tabular}{llllllll}
\hline$a / h$ & Method & $\bar{w}$ & $\bar{\sigma}_{x x}$ & $\bar{\sigma}_{y y}$ & $\bar{\sigma}_{x y}$ & $\bar{\sigma}_{y z}$ & $\bar{\sigma}_{x z}$ \\
\hline 4 & CFS-HSDT [6] & 1.9218 & 0.734 & - & - & 0.183 & - \\
& CFS-TrSDT [50] & 1.9434 & 0.823 & 0.497 & 0.0536 & 0.201 & 0.245 \\
& CFS-ESDT [51] & 1.944 & 0.775 & 0.502 & 0.0516 & 0.191 & 0.220 \\
& CFS-IHSDT [52] & 1.955 & 0.8079 & 0.5015 & 0.0532 & 0.2019 & 0.2438 \\
& Elasticity [47] & 2.006 & 0.755 & 0.556 & 0.0505 & 0.217 & 0.282 \\
& IGA (Arya) & 1.9346 & 0.7562 & 0.5029 & 0.0507 & 0.1877 & 0.2113 \\
& IGA (Soldatos) & 1.9204 & 0.7333 & 0.5023 & 0.0496 & 0.1828 & 0.2016 \\
& IGA (present) & 1.9515 & 0.7955 & 0.5020 & 0.0526 & 0.1974 & 0.2331 \\
& & & & & & & \\
10 & CFS-HSDT [6] & 0.7125 & 0.568 & - & - & 0.103 & - \\
& CFS-TrSDT [50] & 0.7342 & 0.588 & 0.276 & 0.0288 & 0.115 & 0.314 \\
& CFS-ESDT [51] & 0.723 & 0.576 & 0.272 & 0.0281 & 0.108 & 0.272 \\
& CFS-IHSDT [52] & 0.7329 & 0.5845 & 0.2757 & 0.0286 & 0.1148 & 0.3091 \\
& Elasticity [47] & 0.7405 & 0.590 & 0.288 & 0.0289 & 0.123 & 0.357 \\
& IGA (Arya) & 0.7180 & 0.5723 & 0.2705 & 0.0279 & 0.1059 & 0.2583 \\
& IGA (Soldatos) & 0.7120 & 0.5675 & 0.2685 & 0.0276 & 0.1031 & 0.2435 \\
& IGA (present) & 0.7289 & 0.5809 & 0.2740 & 0.0284 & 0.1119 & 0.2924 \\
& & & & & & & \\
20 & CFS-TrSDT [50] & 0.5113 & 0.551 & 0.206 & 0.0233 & 0.090 & 0.331 \\
& CFS-ESDT [51] & 0.508 & 0.548 & 0.205 & 0.0231 & 0.086 & 0.285 \\
& CFS-IHSDT [52] & 0.5102 & 0.5503 & 0.2065 & 0.0233 & 0.0903 & 0.3252 \\
Elasticity [47] & - & 0.552 & 0.210 & 0.0234 & 0.094 & 0.385 \\
& IGA (Arya) & 0.5057 & 0.5464 & 0.2046 & 0.0231 & 0.0842 & 0.2697 \\
& IGA (Soldatos) & 0.5040 & 0.5452 & 0.2039 & 0.0230 & 0.0824 & 0.2536 \\
& IGA (present) & 0.5089 & 0.5487 & 0.2058 & 0.0232 & 0.0883 & 0.3069 \\
& & & & & & & \\
100 & CFS-HSDT [6] & 0.4342 & 0.539 & - & - & 0.075 & - \\
CFS-TrSDT [50] & 0.4353 & 0.539 & 0.181 & 0.0214 & 0.081 & 0.337 \\
CFS-ESDT [51] & 0.435 & 0.538 & 0.18 & 0.0213 & 0.078 & 0.289 \\
CFS-IHSDT [52] & 0.4344 & 0.5392 & 0.1807 & 0.0214 & 0.0813 & 0.3309 \\
Elasticity [47] & - & 0.539 & 0.181 & 0.0213 & 0.083 & 0.395 \\
IGA (Arya) & 0.4343 & 0.5383 & 0.1804 & 0.0213 & 0.0763 & 0.2738 \\
IGA (Soldatos) & 0.4342 & 0.5382 & 0.1803 & 0.0213 & 0.0749 & 0.2572 \\
& IGA (present) & 0.4344 & 0.5384 & 0.1804 & 0.0214 & 0.0796 & 0.3121
\end{tabular}



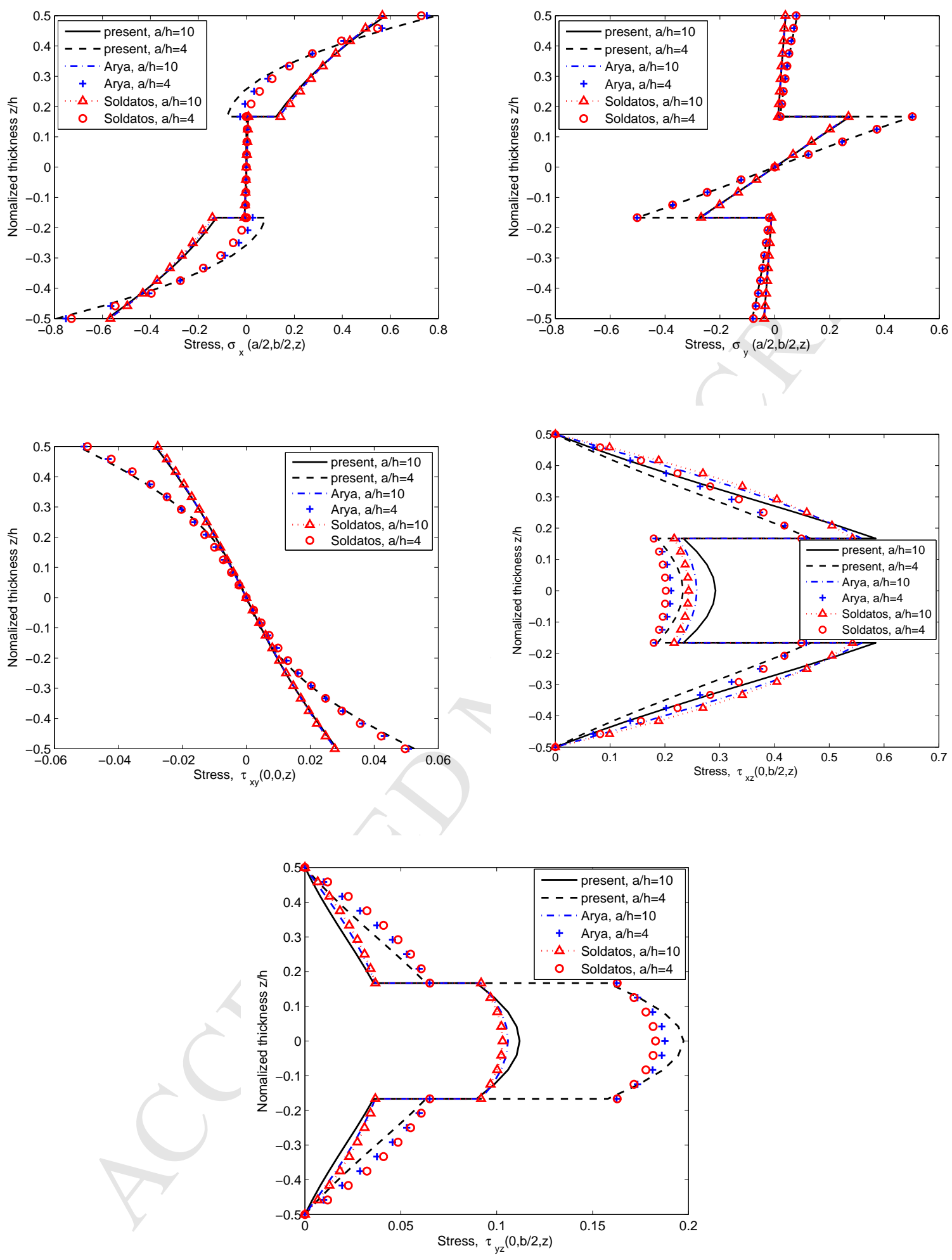

Figure 7: The distribution of stresses through the thickness of three layer $\left[0^{0} / 90^{0} / 0^{0}\right]$ square plate under a sinusoidally distributed load. 
$[38,7]$. It is event that the present HSDT model produce truly the mechanical characterization of the sandwich plates.

\subsubsection{Three-layer sandwich square plate subjected to a uniform load}

Let us consider a simply supported sandwich square plate proposed by Srinivas [53] subjected to a uniform transverse load $q$, as shown in Fig. 9. Length to thickness ratio, $a / h$, is taken as 10. The core thickness $h_{c}$ to face sheet thickness ratio $h_{f}$ is fixed at $8\left(h_{c} / h_{f}=8\right)$. The laminate sandwich plate is made of one inner layer (core), which has the following properties

$$
\bar{Q}_{\text {core }}=\left[\begin{array}{ccccc}
0.999781 & 0.231192 & 0 & 0 & 0 \\
0.231192 & 0.524886 & 0 & 0 & 0 \\
0 & 0 & 0.262931 & 0 & 0 \\
0 & 0 & 0 & 0.266810 & 0 \\
0 & 0 & 0 & 0 & 0.159914
\end{array}\right]
$$

and two outside layers (skins) are calculated as

$$
\bar{Q}_{\text {skin }}=R \bar{Q}_{\text {core }}
$$

The normalized displacement and stresses of the plate are defined as

$$
\begin{gathered}
\bar{w}=0.999781 w\left(\frac{a}{2}, \frac{a}{2}, 0\right) / h q, \quad \bar{\sigma}_{x x}^{1}=\sigma_{x x}^{1}\left(\frac{a}{2}, \frac{a}{2}, \frac{h}{2}\right) / q, \\
\bar{\sigma}_{x x}^{2}=\sigma_{x x}^{1}\left(\frac{a}{2}, \frac{a}{2}, \frac{2 h}{5}\right) / q, \quad \bar{\sigma}_{x x}^{3}=\sigma_{x x}^{2}\left(\frac{a}{2}, \frac{a}{2}, \frac{2 h}{5}\right) / q, \\
\bar{\sigma}_{y y}^{1}=\sigma_{y y}^{1}\left(\frac{a}{2}, \frac{a}{2}, \frac{h}{2}\right) / q, \bar{\sigma}_{y y}^{2}=\sigma_{y y}^{1}\left(\frac{a}{2}, \frac{a}{2}, \frac{2 h}{5}\right) / q, \quad \bar{\sigma}_{y y}^{3}=\sigma_{y y}^{2}\left(\frac{a}{2}, \frac{a}{2}, \frac{2 h}{5}\right) / q .
\end{gathered}
$$

The exact solution of this problem was given by Srinivas [53]. For a comparison, we compute the normalized displacement and stresses of the sandwich square plate using $17 \times 17 \mathrm{~B}$-spline elements. Obtained results from the IGA based on ITSDT are compared with those of the FEM based on HSDT reported by Pandya and Kant [54], the multiquadric radial basis function method (RBFs) based on a finite point formulation and HSDT by Ferreira et al. [49], the multiquadric radial basis function method (RBFs) relied on the layerwise deformation theory (LW) by Ferreira [55], the closed form solution based on IHSDT by Grover et al. [37], the closed form solution based on ESDT by Mantari et al.[36], exact solution by Srinivas [53] and the IGA based on dTrSDTs $[38,7]$. The results with respect to various values of $R(R=5,10,15)$ are given in Table 6 . It is observed that the obtained results from the IGA based on present theory and the dTrSDTs [38, 7] are in close agreement with the exact solution and those solutions for all displacement and stresses.

\subsection{Free vibration analysis}

\subsubsection{Square plates}

\subsubsection{Laminated composite square plate}

Let us consider a four-layer $\left[0^{0} / 90^{0} / 90^{0} / 0^{0}\right]$ plate with simply supported boundary conditions. Material $I I I$ is used. The effects of the length to thickness $a / h$ and elastic modulus ratios $E_{1} / E_{2}$ are studied. To show the convergence of the present approach, the length to thickness $a / h=5$ and elastic modulus ratios $E_{1} / E_{2}=40$ are chosen. As shown in Table 7, the normalized frequency are 
Table 5: The normalized displacement and stresses of a sandwich $\left(0^{0} /\right.$ core $\left./ 0^{0}\right)$ simple supported square plate under a sinusoidally distributed load

\begin{tabular}{|c|c|c|c|c|c|c|c|}
\hline$a / h$ & Method & $\bar{w}$ & $\bar{\sigma}_{x}$ & $\bar{\sigma}_{y}$ & $\bar{\sigma}_{x y}$ & $\bar{\sigma}_{x z}$ & $\bar{\sigma}_{y z}$ \\
\hline \multirow[t]{7}{*}{4} & CFS-HSDT [42] $]^{a}$ & 7.0551 & 1.5137 & 0.2648 & 0.1379 & 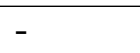 & 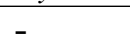 \\
\hline & CFS-HSDT $[42]^{b}$ & 7.0873 & 1.4182 & 0.2365 & 0.1383 & - & - \\
\hline & CFS-FSDT $[42]^{c}$ & 4.7666 & 0.8918 & 0.1562 & 0.0907 & - & - \\
\hline & Elasticity [47] & - & 1.512 & 0.2533 & 0.1437 & - & \\
\hline & IGA (Arya [38]) & 7.0928 & 1.4420 & 0.2379 & 0.1394 & 0.2832 & 0.1211 \\
\hline & IGA (Soldatos [7]) & 7.0849 & 1.4226 & 0.2359 & 0.1381 & 0.2697 & 0.1164 \\
\hline & IGA (present) & 6.9609 & 1.4587 & 0.2378 & 0.1397 & 0.3082 & 0.1283 \\
\hline \multirow[t]{7}{*}{10} & CFS-HSDT [42] $]^{a}$ & 2.0798 & 1.1523 & 0.1100 & 0.0685 & & - \\
\hline & CFS-HSDT $[42]^{b}$ & 2.0629 & 1.1300 & 0.1030 & 0.0679 & & - \\
\hline & CFS-FSDT $[42]^{c}$ & 1.5604 & 1.0457 & 0.0798 & 0.0552 & - & - \\
\hline & Elasticity [47] & - & 1.152 & 0.1099 & 0.0707 & 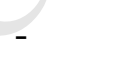 & 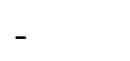 \\
\hline & IGA (Arya [38]) & 2.0681 & 1.1336 & 0.1032 & 0.0682 & 0.3465 & 0.0598 \\
\hline & IGA (Soldatos [7]) & 2.0621 & 1.1296 & 0.1028 & 0.0679 & 0.3287 & 0.0576 \\
\hline & IGA (present) & 2.0465 & 1.1382 & 0.1027 & 0.0680 & 0.3780 & 0.0633 \\
\hline \multirow[t]{7}{*}{20} & CFS-HSDT [42 $]^{a}$ & 1.1933 & 1.1110 & 0.0705 & 0.0504 & - & 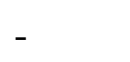 \\
\hline & CFS-HSDT $[42]^{b}$ & 1.1876 & 1.1039 & 0.0679 & 0.0502 & - & 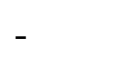 \\
\hline & CFS-FSDT $[42]^{c}$ & 1.0524 & 1.0830 & 0.0612 & 0.0466 & - & - \\
\hline & Elasticity [47] & - & 1.1100 & 0.0700 & 0.0511 & - & 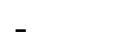 \\
\hline & IGA (Arya [38]) & 1.1891 & 1.1037 & 0.0679 & 0.0502 & 0.3640 & 0.0421 \\
\hline & IGA (Soldatos [7]) & 1.1873 & 1.1026 & 0.0677 & 0.0501 & 0.3451 & 0.0407 \\
\hline & IGA (present) & 1.1835 & 1.1050 & 0.0677 & 0.0501 & 0.3971 & 0.0447 \\
\hline \multirow[t]{7}{*}{50} & CFS-HSDT [42] $]^{a}$ & 0.9296 & 1.1005 & 0.0578 & 0.0445 & - & - \\
\hline & CFS-HSDT $[42]^{b}$ & 0.9284 & 1.0980 & 0.0565 & 0.0445 & - & - \\
\hline & CFS-FSDT $[42]^{c}$ & 0.9063 & 1.0947 & 0.0554 & 0.0439 & - & 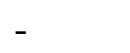 \\
\hline & Elasticity [47] & - & 1.0990 & 0.0569 & 0.0446 & - & - \\
\hline & IGA (Arya [38]) & 0.9286 & 1.0967 & 0.0565 & 0.0445 & 0.3697 & 0.0364 \\
\hline & IGA (Soldatos [7]) & 0.9283 & 1.0965 & 0.0565 & 0.0444 & 0.3504 & 0.0351 \\
\hline & IGA (present) & 0.9277 & 1.0969 & 0.0565 & 0.0444 & 0.4032 & 0.0387 \\
\hline \multirow[t]{7}{*}{100} & CFS-HSDT [42 $]^{a}$ & 0.8913 & 1.0990 & 0.0560 & 0.0436 & - & - \\
\hline & CFS-HSDT [42] $]^{b}$ & 0.8908 & 1.0973 & 0.0549 & 0.0436 & - & - \\
\hline & CFS-FSDT $[42]^{c}$ & 0.8852 & 1.0964 & 0.0546 & 0.0435 & - & 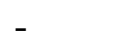 \\
\hline & Elasticity [47] & - & 1.098 & 0.0550 & 0.0437 & - & - \\
\hline & IGA (Arya [38]) & 0.8908 & 1.0957 & 0.0548 & 0.0436 & 0.3705 & 0.0355 \\
\hline & IGA (Soldatos [7]) & 0.8908 & 1.0957 & 0.0548 & 0.0436 & 0.3512 & 0.0343 \\
\hline & IGA (present) & 0.8906 & 1.0958 & 0.0548 & 0.0436 & 0.4041 & 0.0378 \\
\hline
\end{tabular}

${ }^{a} 12$ degrees of freedom per node (DOFs/node); ${ }^{b} 5$ DOFs/node; ${ }^{c} 5$ DOFs/node; 

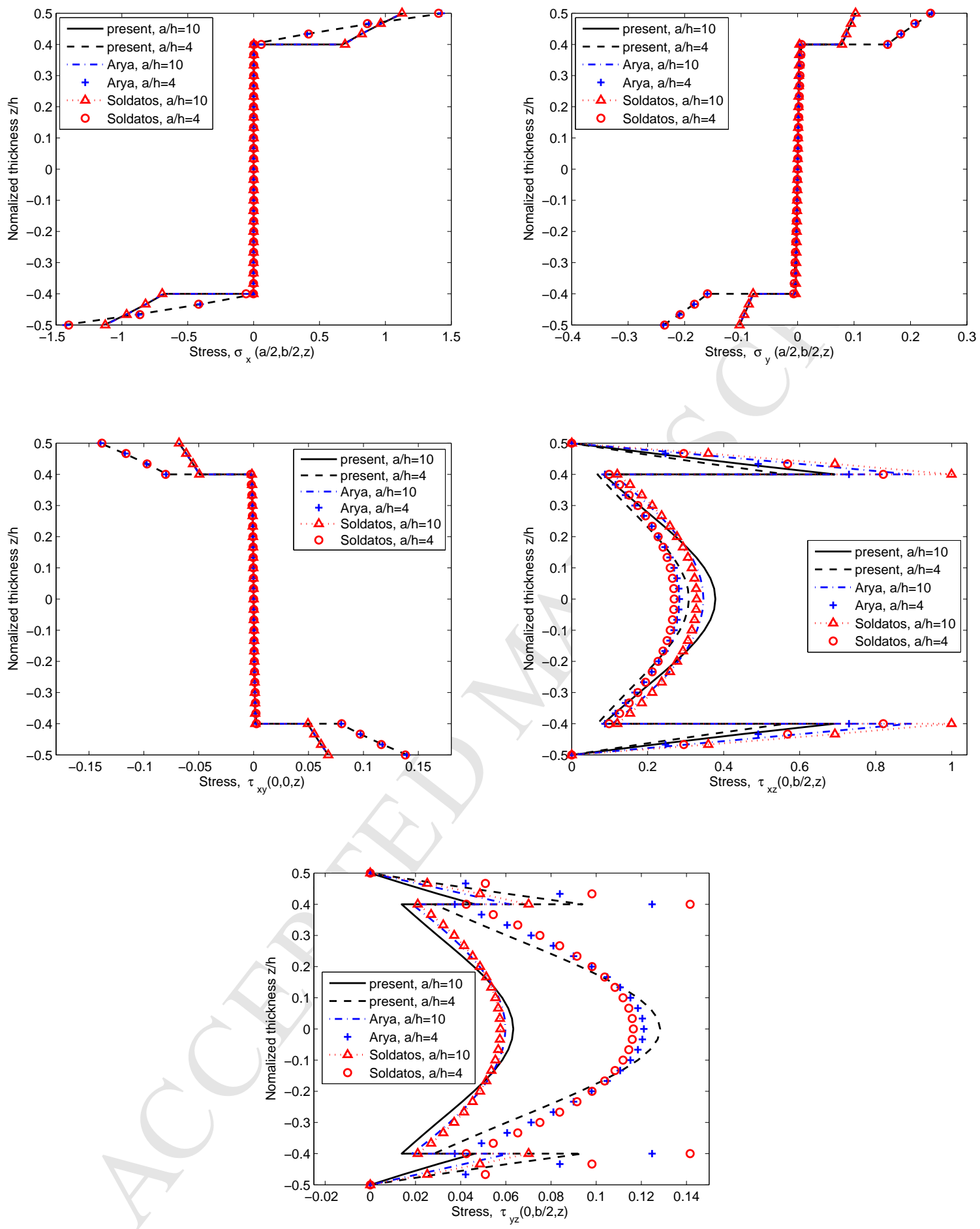

Figure 8: The distribution of stresses through the thickness of a sandwich $\left(0^{0} /\right.$ core $\left./ 0^{0}\right)$ plate under a sinusoidally distributed load. 


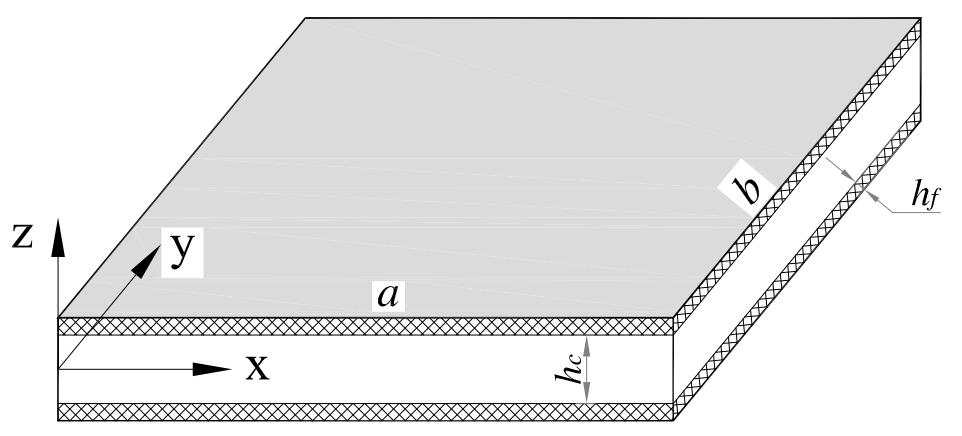

Figure 9: Geometry of a sandwich plate.

Table 6: The normalized displacement and stresses of a square sandwich plate under a uniform load

\begin{tabular}{|c|c|c|c|c|c|c|c|c|}
\hline$R$ & Method & $\bar{w}$ & $\bar{\sigma}_{x}^{1}$ & $\bar{\sigma}_{x}^{2}$ & $\bar{\sigma}_{x}^{3}$ & $\bar{\sigma}_{y}^{1}$ & $\bar{\sigma}_{y}^{2}$ & $\bar{\sigma}_{y}^{3}$ \\
\hline \multirow[t]{9}{*}{5} & FEM-HSDT [54] & 256.130 & 62.380 & 46.910 & 9.382 & 38.930 & 30.330 & 6.065 \\
\hline & RBFs-HSDT [49] & 257.110 & 60.366 & 47.003 & 9.401 & 38.456 & 30.242 & 6.048 \\
\hline & RBFs-Layerwise [56] & 258.179 & 60.063 & 46.393 & 9.279 & 38.364 & 30.029 & 6.006 \\
\hline & CFS-IHSDT [37] & 255.644 & 60.675 & 47.055 & 9.411 & 38.522 & 30.206 & 6.041 \\
\hline & CFS-ESDT [36] & 256.706 & 60.525 & 47.061 & 9.412 & 38.452 & 30.177 & 6.035 \\
\hline & Exact [53] & 258.970 & 60.353 & 46.623 & 9.340 & 38.491 & 30.097 & 6.161 \\
\hline & IGA (Arya [38]) & 256.998 & 60.375 & 46.953 & 9.390 & 38.449 & 30.213 & 6.042 \\
\hline & IGA (Soldatos [7]) & 256.957 & 60.314 & 46.966 & 9.393 & 38.422 & 30.217 & 6.043 \\
\hline & IGA (present) & 256.212 & 60.495 & 46.990 & 9.398 & 38.456 & 30.196 & 6.039 \\
\hline \multirow[t]{9}{*}{10} & FEM-HSDT [54] & 152 & 50 & 51.310 & 5.131 & 42.830 & 33.970 & 3.397 \\
\hline & RBFs-HSDT[49] & 154.658 & 65.381 & 49.973 & 4.997 & 43.240 & 33.637 & 3.364 \\
\hline & RBFs-Layerwise [56] & 158.912 & 64.993 & 48.601 & 4.860 & 43.491 & 33.409 & 3.341 \\
\hline & CFS-IHSDT [37] & 154.550 & 65.741 & 49.798 & 4.979 & 43.400 & 33.556 & 3.356 \\
\hline & CFS-ESDT [36] & 155.498 & 65.542 & 49.708 & 4.971 & 43.385 & 33.591 & 3.359 \\
\hline & Exact [53] & 159.38 & 65.332 & 48.857 & 4.903 & 43.566 & 33.413 & 3.500 \\
\hline & IGA (Arya [38]) & 155.025 & 65.366 & 49.822 & 4.982 & 43.267 & 33.601 & 3.360 \\
\hline & IGA (Soldatos [7]) & 154.439 & 65.306 & 49.926 & 4.993 & 43.183 & 33.597 & 3.360 \\
\hline & IGA (present) & 154.954 & 65.509 & 49.771 & 4.977 & 43.317 & 33.574 & 3.357 \\
\hline \multirow[t]{9}{*}{15} & FEM-HSDT [54] & 110.430 & 66.620 & 51.970 & 3.465 & 44.920 & 35.410 & 2.361 \\
\hline & RBFs-HSDT [49] & 114.644 & 66.919 & 50.323 & 3.355 & 45.623 & 35.167 & 2.345 \\
\hline & RBFs-Layerwise [56] & 121.347 & 66.436 & 48.011 & 3.201 & 46.385 & 34.965 & 2.331 \\
\hline & CFS-IHSDT [37] & 115.820 & 67.272 & 49.813 & 3.321 & 45.967 & 35.088 & 2.339 \\
\hline & CFS-ESDT [36] & 115.919 & 67.185 & 49.769 & 3.318 & 45.910 & 35.081 & 2.339 \\
\hline & Exact [53] & 121.720 & 66.787 & 48.299 & 3.238 & 46.424 & 34.955 & 2.494 \\
\hline & IGA (Arya [38]) & 115.438 & 66.876 & 50.048 & 3.337 & 45.715 & 35.143 & 2.343 \\
\hline & IGA (Soldatos [7]) & 114.400 & 66.837 & 50.280 & 3.352 & 45.548 & 35.120 & 2.341 \\
\hline & IGA (present) & 116.048 & 67.009 & 49.847 & 3.323 & 45.858 & 35.129 & 2.342 \\
\hline
\end{tabular}


Table 7: The convergence of non-dimensional frequency parameter $\Phi=\left(\omega a^{2} / h\right)\left(\rho / E_{2}\right)^{1 / 2}$ of four-layer $\left[0^{0} / 90^{0} / 90^{0} / 0^{0}\right]$ simply supported laminated square plate $(a / h=5)$

\begin{tabular}{llll}
\hline Method & \multicolumn{3}{c}{ Modes } \\
\cline { 2 - 4 } & $9 \times 9$ & $13 \times 13$ & $17 \times 17$ \\
\hline IGA (Arya [38]) & 10.7933 & 10.7931 & 10.7930 \\
IGA (Touratier [39]) & 10.7933 & 10.7931 & 10.7930 \\
IGA (Soldatos [7]) & 10.7876 & 10.7874 & 10.7873 \\
IGA (present) & 10.8430 & 10.8428 & 10.8428
\end{tabular}

computed using meshes of $9 \times 9,13 \times 13$ and $17 \times 17$. It can be observed that the differences of normalized frequencies between meshes of $13 \times 13$ and $17 \times 17$ are not significant. Hence, for a comparison with other methods, a mesh of $13 \times 13$ cubic elements can be chosen.

The first normalized frequency derived from the IGA based on the present theory (ITSDT) is listed in Table 8 corresponding to various modulus ratios $E_{1} / E_{2}$ and $a / h=5$. The obtained results are compared with the closed form solution based on HSDT [57, 58], the moving least squares differential quadrature method (DQM) [43] based on FSDT, the meshfree method using multiquadric radial basis functions (RBFs) [59] \& wavelets function [60] based on FSDT and our isogeometric approach based on dTrSDTs $[38,7]$. It is found that the obtained results from the IGA based on ITSDT and dTrSDTs are in good agreement with the published ones. And the first normalized frequency of the IGA based on ITSDT is slightly larger the IGA based on dTrSDTs $[38,7]$.

The influence of the length to thickness ratios is also considered, as shown in Table 9. The obtained results are compared with those of Zhen and Wanji [61] based on a global-local higher-order theory (GLHOT), Whu and Chen [62] based on a local higher-order theory (LHOT), Matsunaga [63] based on a glocal-local higher-order theory, Cho et al. [64] based on HSDT. As expected, a good agreement with other published solutions is obtained.

\subsubsection{Composite sandwich square plate}

Let us a five-layer $\left(0^{0} / 90^{\circ} /\right.$ core $\left./ 0^{0} / 90^{\circ}\right)$ anti-symmetry sandwich square plate with a simply supported boundary condition. Material $I V$ is used and the plate is modeled by $13 \times 13 \mathrm{~B}$-spline elements. First, the changes of the length to thickness ratio and thickness of core to thickness of face sheet ratio are considered. The normalized frequencies are defined as $\varpi=\left(\omega b^{2} / h\right) \sqrt{\left(\rho / E_{2}\right)_{\text {face }}}$. For various length to thickness ratios varying from 2 to 100 , the first normalized frequency is listed in Table 10. The results obtained are compared with analytical solutions provided in [44] based on HSDT \& FSDT and our isogeometric approach based on dTrSDTs [7, 38]. We observed that present results are in good agreement with analytical ones from HSDT model (12 DOFs/node) reported in [44] and are more accuracy than those of HSDT (5 DOFs/node) \& FSDT (5 DOFs/node) [44]. It is clear that the difference between results of using FSDT model and HSDT model is very 
Table 8: A non-dimensional frequency parameter $\varpi=\left(\omega a^{2} / h\right)\left(\rho / E_{2}\right)^{1 / 2}$ of a $\left[0^{0} / 90^{0} / 90^{0} / 0^{0}\right]$ simply supported laminated square plate $(a / h=5)$

\begin{tabular}{lclll}
\hline Method & \multicolumn{4}{c}{$E_{1} / E_{2}$} \\
\cline { 2 - 5 } & 10 & 20 & 30 & 40 \\
\hline RBFs-FSDT[59] & 8.2526 & 9.4974 & 10.2308 & 10.7329 \\
Wavelets-FSDT[60] & 8.2794 & 9.5375 & 10.2889 & 10.8117 \\
DQM-FSDT [43] & 8.2924 & 9.5613 & 10.3200 & 10.8490 \\
CFS-HSDT[58, 57] & 8.2982 & 9.5671 & 10.3260 & 10.8540 \\
IGA (Arya [38]) & 8.2737 & 9.5302 & 10.2769 & 10.7931 \\
IGA (Soldatos [7]) & 8.2719 & 9.5263 & 10.2719 & 10.7874 \\
IGA (present) & 8.2944 & 9.5650 & 10.3206 & 10.8428
\end{tabular}

Table 9: A non-dimensional frequency parameter $\varpi=\left(\omega a^{2} / h\right)\left(\rho / E_{2}\right)^{1 / 2}$ of a $\left[0^{0} / 90^{0} / 90^{0} / 0^{0}\right]$ simply supported laminated square plate $\left(E_{1} / E_{2}=40\right)$

\begin{tabular}{llllllll}
\hline Method/Authors & \multicolumn{7}{c}{$a / h$} \\
\cline { 2 - 8 } & 4 & 5 & 10 & 20 & 25 & 50 & 100 \\
\hline Zhen and Wanji [61] & 9.2406 & 10.7294 & 15.1658 & 17.8035 & 18.2404 & 18.9022 & 19.1566 \\
Whu and Chen [62] & 9.193 & 10.682 & 15.069 & 17.636 & 18.055 & 18.670 & 18.835 \\
Matsunaga [63] & 9.1988 & 10.6876 & 15.0721 & 17.6369 & 18.0557 & 18.6702 & 18.8352 \\
Cho et al. [64] & - & 10.673 & 15.066 & 17.535 & 18.054 & 18.670 & 18.835 \\
IGA (Arya [38]) & 9.3295 & 10.7931 & 15.1130 & 17.6492 & 18.0638 & 18.6724 & 18.8358 \\
IGA (Soldatos [7]) & 9.3236 & 10.7874 & 16.8498 & 17.6465 & 18.0619 & 18.6718 & 18.8357 \\
IGA (present) & 9.3781 & 10.8428 & 15.1552 & 17.6677 & 18.0766 & 18.6760 & 18.8367 \\
& & & & & & & \\
\hline
\end{tabular}


Table 10: The first normalized frequency $\varpi=\left(\omega b^{2} / h\right) \sqrt{\left(\rho / E_{2}\right)_{f}}$ of an antisymmetry $\left(0^{0} / 90^{0} /\right.$ core $\left./ 0^{0} / 90^{0}\right)$ sandwich square plate with $h_{c} / h_{f}=10$

\begin{tabular}{lllllll}
\hline$a / h$ & HSDT [44] $^{a}$ & HSDT [44] $^{b}$ & FSDT [44] $^{c}$ & IGA [38] & IGA [7] & IGA (present) \\
\hline 2 & 1.1941 & 1.6252 & 5.2017 & 1.4858 & 1.6806 & 1.2133 \\
4 & 2.1036 & 3.1013 & 9.0312 & 2.8122 & 3.2115 & 2.2298 \\
10 & 4.8594 & 7.0473 & 13.8694 & 6.4640 & 7.2607 & 5.2038 \\
20 & 8.5955 & 11.2664 & 15.5295 & 10.6397 & 11.4827 & 9.0830 \\
30 & 11.0981 & 13.6640 & 15.9155 & 12.8874 & 13.5273 & 11.5583 \\
40 & 12.6821 & 14.430 & 16.0577 & 14.0938 & 14.5536 & 13.0645 \\
50 & 13.6899 & 15.0323 & 16.1264 & 14.7811 & 15.1159 & 13.9958 \\
60 & 14.3497 & 15.3868 & 16.1612 & 15.1997 & 15.4503 & 14.5939 \\
70 & 14.7977 & 15.6134 & 16.1845 & 15.4701 & 15.6631 & 14.9940 \\
80 & 15.1119 & 15.7660 & 16.1991 & 15.6536 & 15.8061 & 15.2721 \\
90 & 15.3380 & 15.8724 & 16.2077 & 15.7832 & 15.9064 & 15.4720 \\
100 & 15.5093 & 15.9522 & 16.2175 & 15.8780 & 15.9794 & 15.6200 \\
& & & & & & \\
\hline
\end{tabular}

${ }^{a} 12 \mathrm{DOF} /$ node; $^{b} 5$ DOFs/node; ${ }^{c} 5$ DOFs/node;

significant for thick sandwich plates. It is therefore necessary to use HSDT model. Table 11 lists several higher frequencies for moderately thick and thin plates. It can be found that present results agree well with those obtained by HSDT models for both thick $(a / h=10)$ and thin $(a / h=100)$ sandwich plate whereas FSDT model leads to over-stiffness of the natural frequencies. The first six mode shapes are illustrated in Fig. 10.

Next, the influence of the thickness of core to the thickness of face sheet ratio $h_{c} / h_{f}$ on the natural normalized frequency is resulted in Table 12. For the range of $h_{c} / h_{f}$ from 4 to 100 , The natural frequency values based on FSDT model [65] are very high compared to the results based on HSDT model [65]. As expected, the present method is in good agreement with the analytical solution based on HSDT [65] (12 DOFs/node) and is also more accuracy than that of HSDT [65] (5 DOFs/node). The natural frequencies of the plate decrease as the ratio $h_{c} / h_{f}$ increases, i.e, the stiffness of the plate decreases.

\subsubsection{Circular plates}

A circular four-layer $[\theta /-\theta /-\theta / \theta]$ laminated plate with fully clamped boundary and various fibre orientation angles $\alpha=0^{0} ; 15^{0} ; 30^{0} ; 45^{0}$ are illustrated in Fig. 11a. Material parameter III is used. The circular plate has a radius to thickness ratio of $5(R / h=5)$. For this problem, a NURBS quadratic basis function is enough to model exactly the circular geometry. Knot vectors $\Xi \times \mathscr{H}$ of the coarsest mesh with one element are defined as follows $\Xi=$ $\{0,0,0,1,1,1\} ; \mathscr{H}=\{0,0,0,1,1,1\}$. Data of the circular plate are given in Table 13. Coarse mesh and control net of the plate with respect to quadratic and cubic elements are displayed in Fig. 12. Fig. $11 \mathrm{~b}$ describes $13 \times 13$ NURBS cubic elements. The normalized frequencies are defined as $\varpi=\left(4 \omega R^{2} / h\right)\left(\rho / E_{2}\right)^{1 / 2}$ 
Table 11: Normalized frequencies $\varpi=\left(\omega b^{2} / h\right) \sqrt{\left(\rho / E_{2}\right)_{f}}$ of an antisymmetry $\left(0^{0} / 90^{0} /\right.$ core $\left./ 0^{0} / 90^{0}\right)$ sandwich simply supported square plate with $h_{c} / h_{f}=10$

\begin{tabular}{|c|c|c|c|c|c|c|c|}
\hline \multirow[t]{2}{*}{$a / h$} & \multirow[t]{2}{*}{ Method } & \multicolumn{6}{|c|}{ Modes } \\
\hline & & 1 & 2 & 3 & 4 & 5 & 6 \\
\hline \multirow[t]{6}{*}{10} & $\operatorname{HSDT}[65]^{a}$ & 4.8594 & 8.0187 & 10.2966 & 11.7381 & 13.4706 & 16.1320 \\
\hline & $\operatorname{HSDT}[65]^{b}$ & 7.0473 & 11.9087 & 15.2897 & 17.3211 & 19.8121 & 23.5067 \\
\hline & FSDT $[65]^{c}$ & 13.8694 & 30.6432 & 41.5577 & 50.9389 & 58.3636 & 71.3722 \\
\hline & IGA (Arya [38]) & 6.4640 & 10.8249 & 13.8794 & 15.8230 & 18.0725 & 21.4991 \\
\hline & IGA (Soldatos [7]) & 7.2607 & 12.3233 & 15.8342 & 18.0383 & 20.6086 & 22.5448 \\
\hline & IGA (present) & 5.2038 & 8.5847 & 10.9922 & 12.5963 & 14.3865 & 17.1818 \\
\hline \multirow[t]{6}{*}{100} & $\operatorname{HSDT}[65]^{a}$ & 15.5093 & 39.0293 & 54.7618 & 72.7572 & 83.4412 & 105.3781 \\
\hline & $\operatorname{HSDT}[65]^{b}$ & 15.9521 & 42.2271 & 60.1272 & 83.9982 & 96.3132 & 124.2047 \\
\hline & $\mathrm{FSDT}[65]^{c}$ & 16.2175 & 44.7072 & 64.5044 & 94.9097 & 108.9049 & 143.7969 \\
\hline & IGA (Arya [38]) & 15.8780 & 41.6412 & 59.1265 & 81.7715 & 93.7548 & 120.3777 \\
\hline & IGA (Soldatos [7]) & 15.9794 & 42.4265 & 60.4657 & 84.7776 & 97.2019 & 125.5390 \\
\hline & IGA (present) & 15.6200 & 39.7699 & 55.9857 & 75.1842 & 86.2115 & 109.3430 \\
\hline
\end{tabular}

${ }^{a} 12 \mathrm{DOF} /$ node; ${ }^{b} 5 \mathrm{DOFs} /$ node; ${ }^{c} 5$ DOFs/node;

Table 12: The first normalized frequency $\varpi=\left(\omega b^{2} / h\right) \sqrt{\left(\rho / E_{2}\right)_{f}}$ of an antisymmetry $\left(0^{0} / 90^{0} /\right.$ core $\left./ 0^{0} / 90^{0}\right)$ sandwich square plate with $a / h=10$

\begin{tabular}{lllllll}
\hline$t_{c} / t_{f}$ & HSDT [65] $^{a}$ & HSDT [65] & FSDT [65] & IGA [38] & IGA [7] & IGA (present) \\
\hline 4 & 8.9948 & 10.7409 & 13.9190 & 10.3446 & 10.9424 & 9.0883 \\
10 & 4.8594 & 7.0473 & 13.8694 & 6.4640 & 7.2607 & 5.2038 \\
20 & 3.1435 & 4.3734 & 12.8946 & 4.0052 & 4.5010 & 3.3853 \\
30 & 2.8481 & 3.4815 & 11.9760 & 3.2876 & 3.5534 & 3.0179 \\
40 & 2.8266 & 3.1664 & 11.2036 & 3.0706 & 3.2072 & 2.9676 \\
50 & 2.8625 & 3.0561 & 10.5557 & 3.0137 & 3.0803 & 2.9925 \\
100 & 3.0290 & 3.0500 & 8.4349 & 3.0781 & 3.0537 & 3.1536
\end{tabular}

${ }^{a} 12 \mathrm{DOFs} /$ node; ${ }^{b} 5 \mathrm{DOFs} /$ node; ${ }^{c} 5 \mathrm{DOFs} /$ node;

Table 13: Control points and weights for a circular plate with radius $R=0.5$

\begin{tabular}{cccccccccc}
\hline $\mathrm{i}$ & 1 & 2 & 3 & 4 & 5 & 6 & 7 & 8 & 9 \\
\hline$x_{i}$ & $-\sqrt{2} / 4$ & $-\sqrt{2} / 2$ & $-\sqrt{2} / 4$ & 0 & 0 & 0 & $\sqrt{2} / 4$ & $\sqrt{2} / 2$ & $\sqrt{2} / 4$ \\
\hline$y_{i}$ & $\sqrt{2} / 4$ & 0 & $-\sqrt{2} / 4$ & $\sqrt{2} / 2$ & 0 & $-\sqrt{2} / 2$ & $\sqrt{2} / 4$ & 0 & $-\sqrt{2} / 4$ \\
\hline$w_{i}$ & 1 & $\sqrt{2} / 2$ & 1 & $\sqrt{2} / 2$ & 1 & $\sqrt{2} / 2$ & 1 & $\sqrt{2} / 2$ & 1 \\
\hline
\end{tabular}



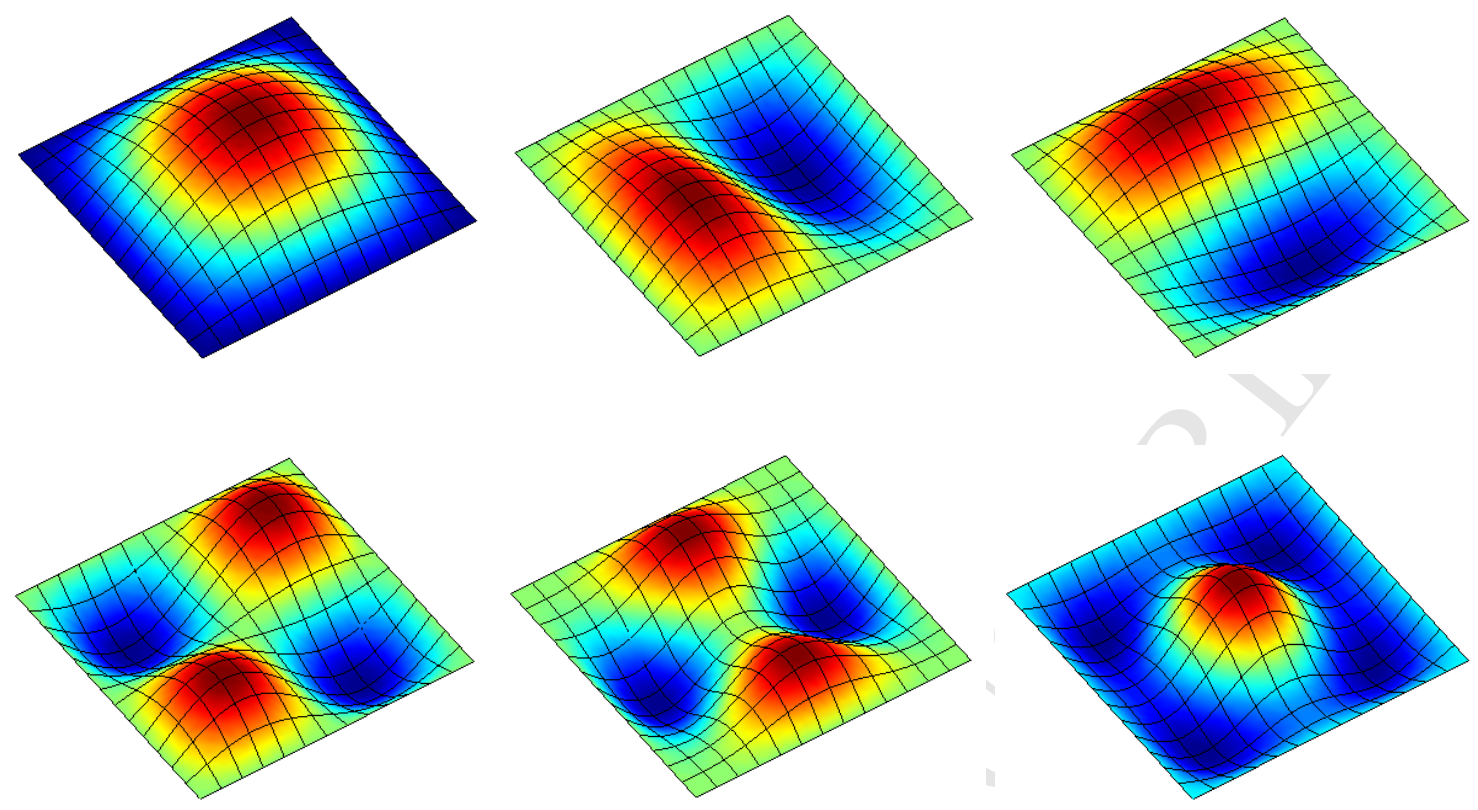

Figure 10: First six mode shapes of antisymmetry the sandwich $\left(0^{0} / 90^{\circ} /\right.$ core $\left./ 0^{0} / 90^{\circ}\right)$ simply supported square plate.

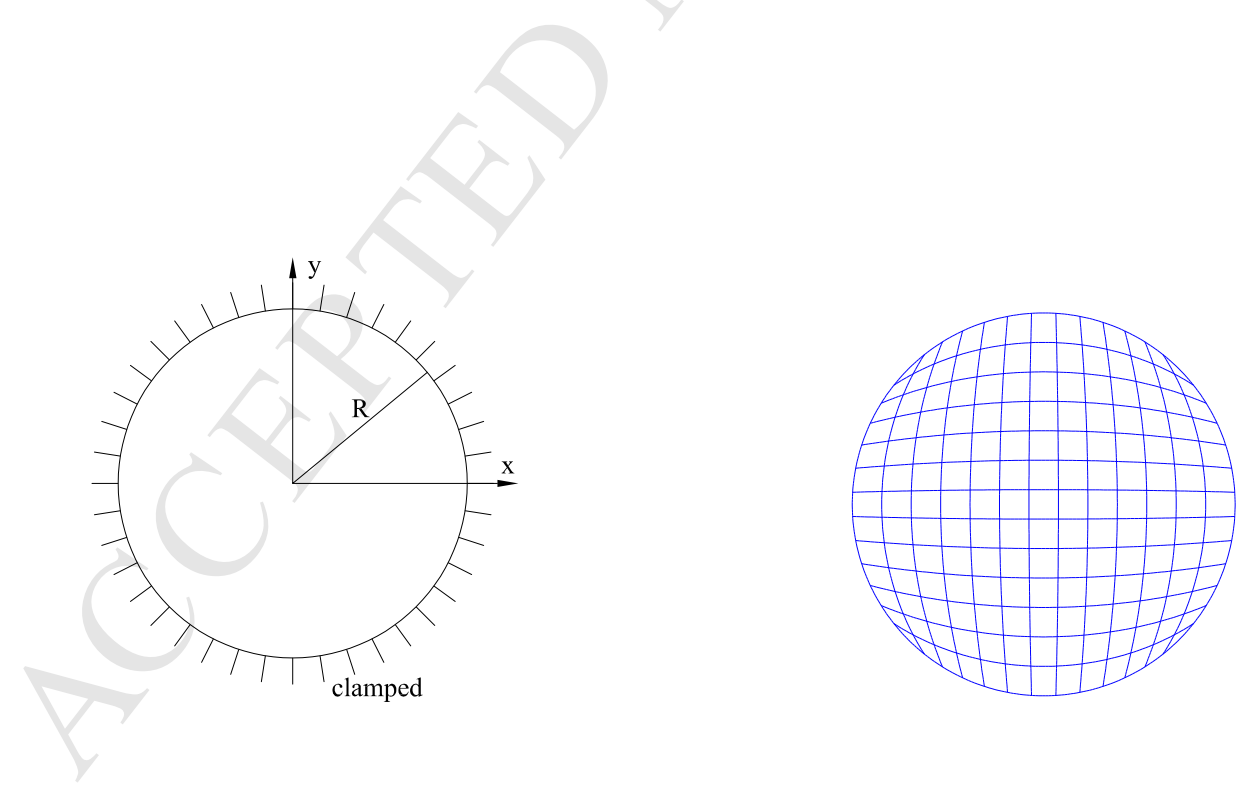

Figure 11: Geometry and element mesh of a circular plate 


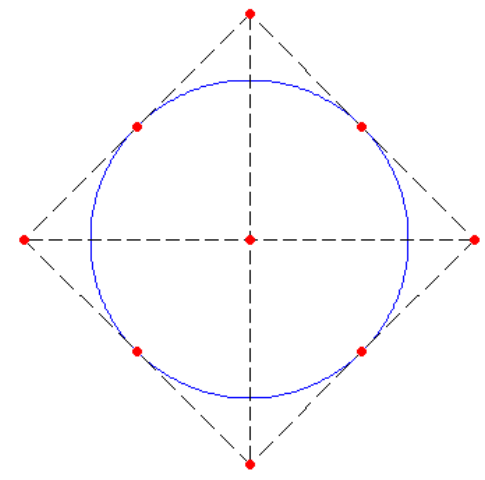

(a)

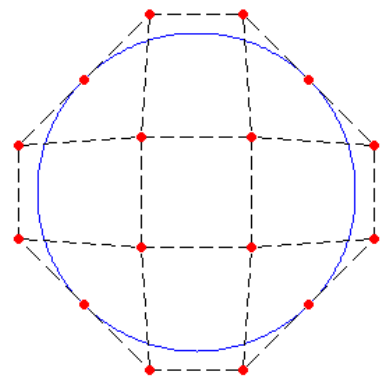

(b)

Figure 12: Coarse mesh and control points of a circular plate with various degrees: a) $p=2$; and b) $p=3$.

Table 14 exhibits the first six normalized frequencies derived from the present approach in comparison with the moving least squares differential quadrature method (MLSDQ) based on FSDT [43] and the isogeometric approach based on dTrSDTs [38, 7]. A good agreement is obtained for the present model. Fig. 13 provides the first six mode shapes of a circular four-layer $[45 /-45 /-45 / 45]$ clamped laminated plate. It is observed that mode shapes are very smooth due to using NURBS basis functions.

\subsubsection{Elliptical plates}

Let us consider a three layer $\left[0^{0} / 90^{0} / 0^{0}\right]$ elliptical plate with a fully clamped boundary using Material $V$. The elliptical plate radiuses are $a=5$ and $b=2.5$ as plotted in Fig. 14, respectively. The normalized frequency is defined by $\varpi=\left(\omega a^{2}\right)\left(\rho h / D_{0}\right)^{1 / 2}$ with $D_{0}=E_{2} h^{3} / 12\left(1-v_{12} v_{21}\right)$. Numerical study for this problem was addressed by Chen et al. [45] using the Element Free Galerkin method (EFG) and classical laminated plate theory (CLPT). The normalized first six frequencies with various the length to thickness ratios using the present theory and the dTrSDTs $[38,7]$ are provided in Table 15 . The present solution is in good agreement with other ones. For the thin case $a / h=100$, the present solutions outperform the EFG ones. The first six mode shapes of three-layer $\left[0^{0} / 90^{0} / 0^{0}\right]$ fully clamped laminated elliptical plate are depicted in Fig. 15.

\subsection{Buckling analysis of laminated composite and sandwich plates}

For a comparison, the buckling load factor is normalized as $\bar{\lambda}=\lambda_{c r} a^{2} /\left(E_{2} h^{3}\right)$ where $E_{2}$ and $\lambda_{c r}$ are the elastic modulus and the critical buckling load, respectively. The plate is modeled by $13 \times 13$ cubic elements for all examples.

\subsubsection{Square plate under uniaxial compression}

\subsubsection{Laminate composite plates}

A simply supported four-layer cross-ply $\left[0^{0} / 90^{0} / 90^{0} / 0^{0}\right]$ square laminated plate is subjected to 
Table 14: A non-dimensional frequencies parameter $\varpi=\left(\omega a^{2} / h\right)\left(\rho / E_{2}\right)^{1 / 2}$ of a circular 4-layer $\left[\theta^{0} /-\theta^{0} /-\theta^{0} / \theta^{0}\right]$ clamped laminated plate

\begin{tabular}{|c|c|c|c|c|c|c|c|}
\hline \multirow[t]{2}{*}{$\theta^{0}$} & \multirow[t]{2}{*}{ Method } & \multicolumn{6}{|c|}{ Mode } \\
\hline & & 1 & 2 & 3 & 4 & 5 & 6 \\
\hline \multirow[t]{4}{*}{$0^{0}$} & MLSDQ-FSDT[43] & 22.211 & 29.651 & 41.101 & 42.635 & 50.309 & 54.553 \\
\hline & IGA (Arya [38]) & 23.2512 & 30.4528 & 41.6978 & 46.4646 & 54.0781 & 56.0772 \\
\hline & IGA (Soldatos [7]) & 23.1297 & 30.3199 & 41.5644 & 46.0359 & 53.5988 & 55.9329 \\
\hline & IGA (present) & 23.5781 & 30.7459 & 42.0042 & 47.6230 & 55.2998 & 56.4994 \\
\hline \multirow[t]{4}{*}{$15^{0}$} & MLSDQ-FSDT[43] & 22.774 & 31.455 & 43.350 & 43.469 & 52.872 & 57.386 \\
\hline & IGA (Arya [38]) & 23.3918 & 31.6349 & 43.8039 & 46.2672 & 55.0467 & 58.6859 \\
\hline & IGA (Soldatos [7]) & 23.3081 & 31.5783 & 43.7640 & 45.9649 & 54.7980 & 58.6459 \\
\hline & IGA (present) & 23.6090 & 31.7743 & 43.9569 & 47.1157 & 55.7728 & 58.9242 \\
\hline \multirow[t]{4}{*}{$30^{0}$} & MLSDQ-FSDT[43] & 24.071 & 36.153 & 43.968 & 51.074 & 56.315 & 66.220 \\
\hline & IGA (Arya [38]) & 24.1128 & 35.6423 & 45.9183 & 50.5740 & 57.4795 & 67.4411 \\
\hline & IGA (Soldatos [7]) & 24.0628 & 35.6831 & 45.6918 & 50.6956 & 57.3925 & 67.6447 \\
\hline & IGA (present) & 24.2081 & 35.6047 & 46.5406 & 50.4242 & 57.8970 & 67.2431 \\
\hline \multirow[t]{4}{*}{$45^{0}$} & MLSDQ-FSDT[43] & 24.752 & 39.181 & 43.607 & 56.759 & 56.967 & 65.571 \\
\hline & IGA (Arya [38]) & 24.5976 & 38.0569 & 45.6121 & 54.6742 & 58.5467 & 69.9105 \\
\hline & IGA (Soldatos [7]) & 24.5566 & 38.1730 & 45.3601 & 54.9789 & 58.4513 & 69.3199 \\
\hline & IGA (present) & 24.6607 & 37.8980 & 46.2506 & 54.2043 & 59.0173 & 71.3684 \\
\hline
\end{tabular}



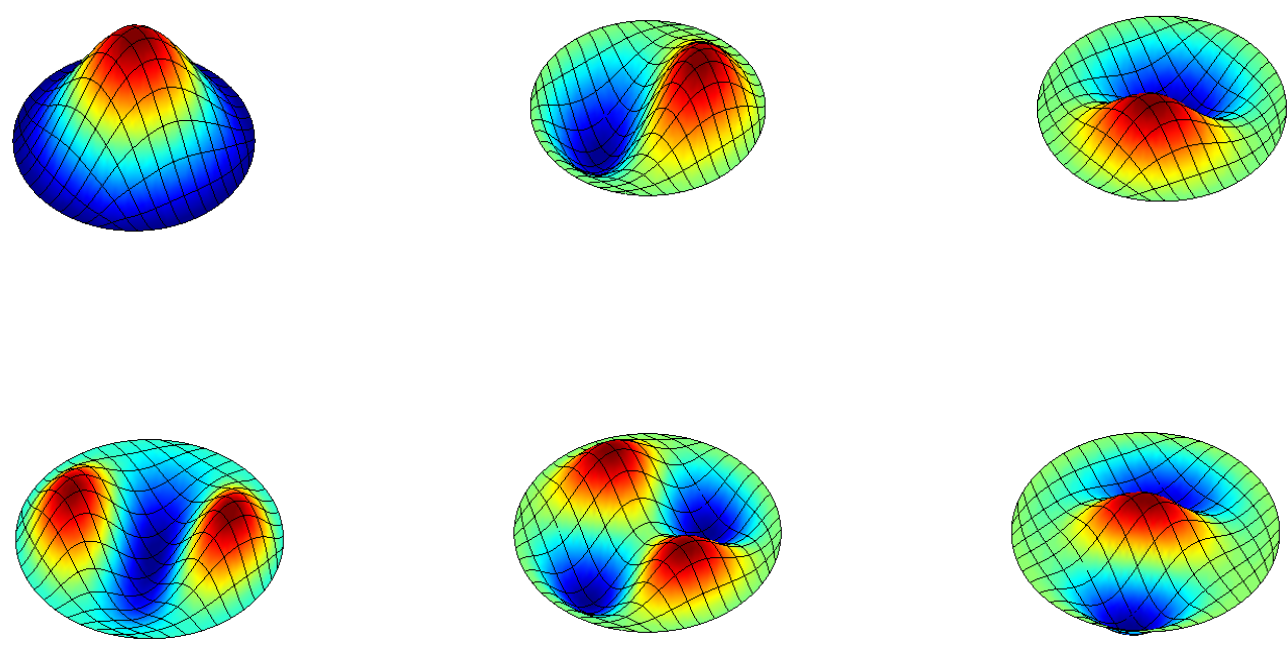

Figure 13: Six mode shapes of a four-layer $\left[45^{0} /-45^{0} /-45^{0} / 45^{0}\right]$ clamped laminated circular plate with $R / h=5$.
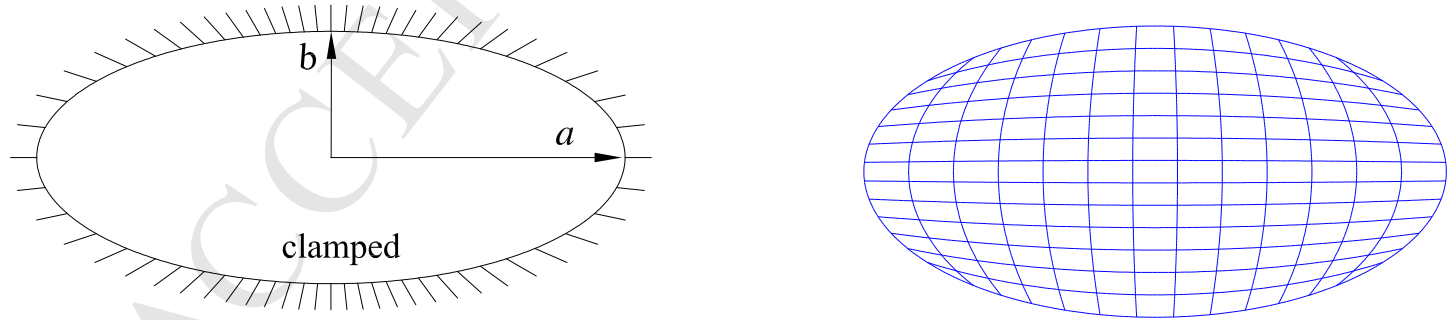

Figure 14: Geometry and element mesh of a clamped elliptical plate. 
Table 15: A non-dimensional frequencies parameter $\varpi=\left(\omega a^{2}\right)\left(\rho h / D_{0}\right)^{1 / 2}$ of a $\left[0^{0} / 90^{0} / 0^{0}\right]$ clamped laminated elliptical plate

\begin{tabular}{|c|c|c|c|c|c|c|c|}
\hline \multirow[t]{2}{*}{$a / h$} & \multirow[t]{2}{*}{ Method } & \multicolumn{6}{|c|}{ Modes } \\
\hline & & 1 & 2 & 3 & 4 & 5 & 6 \\
\hline \multirow[t]{3}{*}{5} & IGA (Arya [38]) & 14.5198 & 20.6664 & 28.1508 & 29.9875 & 36.4140 & 36.4546 \\
\hline & IGA (Soldatos [7]) & 14.4734 & 20.6419 & 28.1647 & 29.8046 & 36.2748 & 36.4721 \\
\hline & IGA (present) & 14.6407 & 20.7592 & 28.1961 & 30.4532 & 36.4321 & 36.8598 \\
\hline \multirow[t]{3}{*}{10} & IGA (Arya [38]) & 17.3531 & 26.1481 & 37.7547 & 39.7467 & 50.1004 & 51.4236 \\
\hline & IGA (Soldatos [7]) & 17.3350 & 26.1460 & 37.7937 & 39.6529 & 50.0147 & 51.5283 \\
\hline & IGA (present) & 17.4003 & 26.1718 & 37.7157 & 39.9878 & 50.3411 & 51.2958 \\
\hline \multirow[t]{3}{*}{20} & IGA (Arya [38]) & 18.4166 & 28.5280 & 42.6786 & 44.5207 & 57.5495 & 60.4237 \\
\hline & IGA (Soldatos [7]) & 18.4113 & 28.5286 & 42.6975 & 44.4885 & 57.5209 & 60.4782 \\
\hline & IGA (present) & 18.4305 & 28.5333 & 42.6563 & 44.6033 & 57.6329 & 60.3551 \\
\hline \multirow[t]{4}{*}{100} & EFG (CLPT) [45] & 18.81 & 29.58 & 44.99 & 46.72 & 61.34 & 65.14 \\
\hline & IGA (Arya [38]) & 18.8106 & 29.4710 & 44.8189 & 46.5396 & 60.9225 & 64.7702 \\
\hline & IGA (Soldatos [7]) & 18.8103 & 29.4711 & 44.8199 & 46.5381 & 60.9212 & 64.7732 \\
\hline & IGA (present) & 18.8113 & 29.4718 & 44.8216 & 46.5445 & 60.9286 & 64.7845 \\
\hline
\end{tabular}
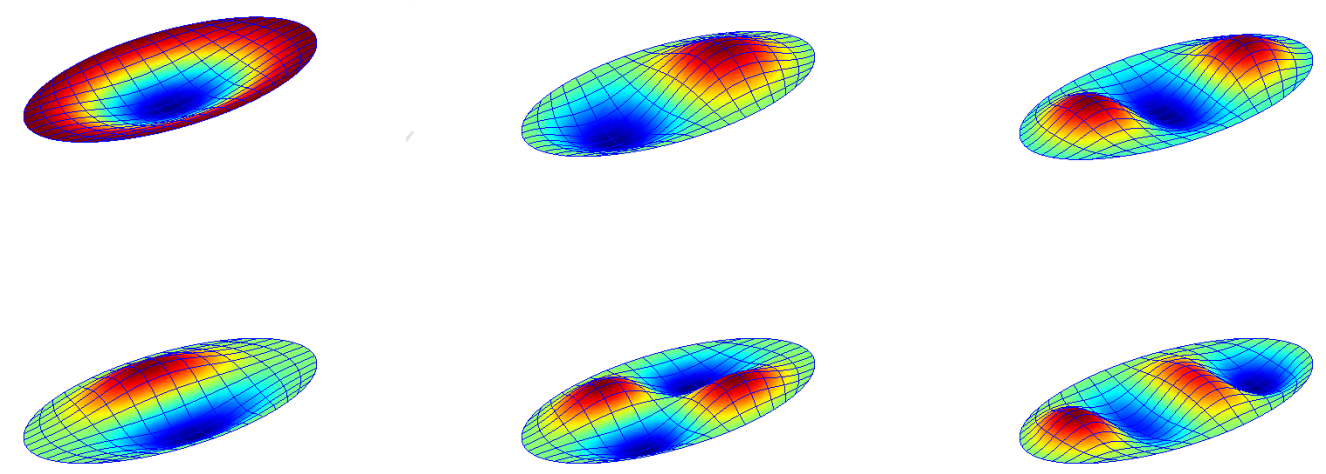

Figure 15: Six mode shapes of a four-layer $\left[0^{0} / 90^{0} / 0^{0}\right]$ clamped laminated ellipse plate with $a / h=10$. 
Table 16: Normalized critical buckling load of simply supported cross-ply $\left[0^{0} / 90^{0} / 90^{0} / 0^{0}\right]$ square plate with various $E_{1} / E_{2}$ ratios and $a / h=10$

\begin{tabular}{llllll}
\hline Method & \multicolumn{5}{c}{$E_{1} / E_{2}$} \\
\cline { 2 - 6 } & 3 & 10 & 20 & 30 & 40 \\
\hline RPIM-HSDT [67] & 5.412 & 10.013 & 15.309 & 19.778 & 23.412 \\
FEM-HSDT [68] & 5.114 & 9.774 & 15.298 & 19.957 & 23.340 \\
FEM-HSDT [69] & 5.442 & 10.026 & 15.418 & 19.813 & 23.489 \\
Elasticity [66] & 5.294 & 9.762 & 15.019 & 19.304 & 22.881 \\
IGA (Arya [38]) & 5.3846 & 9.9120 & 15.2324 & 19.5654 & 23.1858 \\
IGA (Soldatos [7]) & 5.3846 & 9.9106 & 15.2264 & 19.5537 & 23.1682 \\
IGA (present) & 5.3884 & 9.9303 & 15.2841 & 19.6558 & 23.3152 \\
\hline
\end{tabular}

uniaxial compression as shown in Fig. 16a. Material $I I I$ is used. The efficiency and accuracy of the present model for various elastic modulus are investigated. For $a / h=10$ and various elastic modulus $E_{1} / E_{2}$ ratios, the obtained results are compared to the 3D elasticity solution[66], the radial point interpolation method (RPIM) based on HSDT [67] and FEM solution based on HSDT $[68,69]$ and the IGA based on the dTrSDTs [38, 7], as reported in Table 16. It is observed that the IGA based on present theoy is a good competitor with other methods. Also, the normalized critical buckling loads are increased as the $E_{1} / E_{2}$ modulus ratios increase.

In addition, the effect of the length-to-thickness ratios $(a / h)$ subjected to the uniaxial compression load is considered for four layer $\left[0^{0} / 90^{0} / 90^{\circ} / 0^{0}\right]$ simply supported square plates. Table 17 summarizes the normalized critical buckling load of the present method and other methods such as the FEM based on FSDT [70], the FEM based on FSDT [71] \& HSDT [71] and the IGA based on the dTrSDTs $[38,7]$. The present results match well those methods.
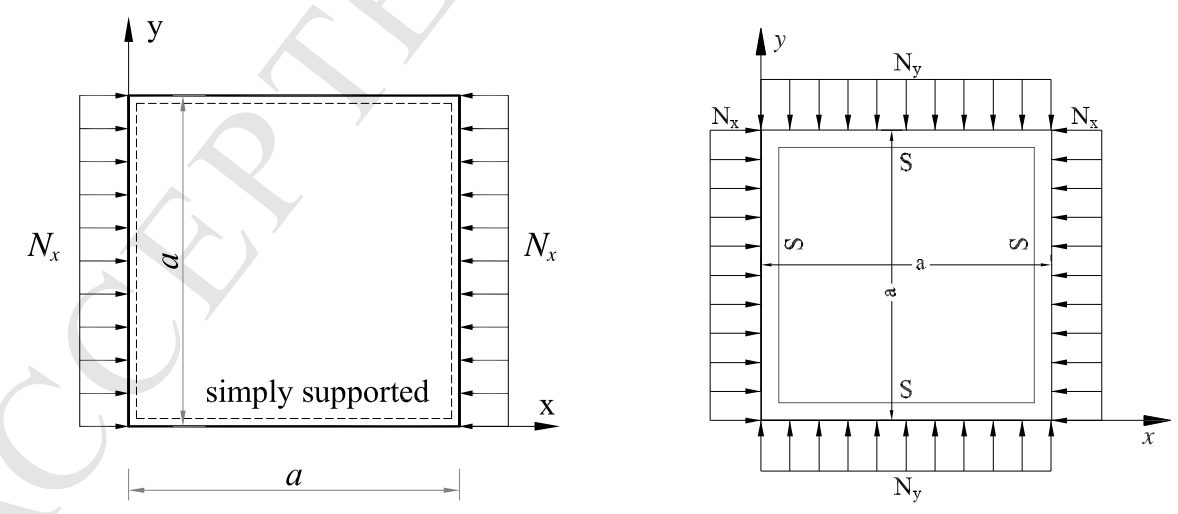

Figure 16: Geometry of laminated composite plates under axial and biaxial compression.

\subsubsection{Composite sandwich plates}

Consider an eleven-layer $\left(0^{0} / 90^{0} / 0^{0} / 90^{0} / 0^{0} /\right.$ core $\left./ 0^{0} / 90^{0} / 0^{0} / 90^{\circ} / 0^{0}\right)$ simply supported sandwich square plate under uniaxial compression, as shown in Fig. 16a. Material VI is used. The 
Table 17: Normalized critical buckling load of simply supported cross-ply $\left[0^{0} / 90^{0} / 90^{0} / 0^{0}\right]$ square plate with various ratios $a / h$ and $E_{1} / E_{2}=40$

\begin{tabular}{lllll}
\hline Method & \multicolumn{4}{c}{$a / h$} \\
\cline { 2 - 5 } & 10 & 20 & 50 & 100 \\
\hline FEM-FSDT [70] & 23.409 & 31.625 & 35.254 & 35.851 \\
FEM-FSDT [71] & 23.471 & 31.707 & 35.356 & 35.955 \\
FEM-HSDT [71] & 23.349 & 31.637 & 35.419 & 35.971 \\
IGA (Arya [38]) & 23.1858 & 31.6313 & 35.3458 & 35.9529 \\
IGA (Soldatos [7]) & 23.1682 & 31.6218 & 35.3438 & 35.9524 \\
IGA (present) & 23.3152 & 31.6975 & 35.3595 & 35.9565 \\
\hline
\end{tabular}

length-to-thickness ratio of the plate $a / h$ is taken to be 10 and 20. Various thickness of face sheet to thickness of plate ratios are studied. Table 18 consists of the results derived from the present approach with the IGA based on dTrSDTs [38, 7], the 3D elasticity solution [72], finite element solutions based on HSDT \& FSDT [46] and finite element solution/closed form solution (CFS) based on layerwise (LW) theory [73]. It is again seen that results of the present ITSDT model match well with those of dTrSDTs $[38,7]$ models and are slightly more accurate that those of several above mentioned methods. Note that the FE solution based on FSDT [46] is only reasonable for the sandwich plate with thin face sheet $t_{f} / h<0.075$. For a larger size of face sheet, the FSDT results are significantly deviated.

Next, a symmetric twenty-one layer $\left(\left[0^{0} / 90^{0}\right]_{5} /\right.$ core $\left./\left[90^{0} / 0^{0}\right]_{5}\right)$ sandwich square plate with a simply supported boundary condition is considered. The sandwich plate includes 10 layers of cross-ply laminated face sheets of equal thickness and a soft orthotropic core. The buckling load factor with respect to various parameters like the length to thickness $(a / h)$ and thickness of face sheet to thickness of plate $\left(h_{f} / h\right)$ ratios are presented. The results obtained are compared with those of several available solutions such as 3D elasticity solution by Noor et al. [72], closed form solution based on HSDT by Dafedar et al.[74], finite element solution based on high-order globallocal plate theory (GLPT) by Shariyat [75], closed form solution based on mixed layerwise (MLW) theory by Dafedar et al. [74], closed form solution based on HSDT by Kheirikhaha et al.[76] and the IGA based on dTrSDTs [38, 7], as listed in Table 18. As expected, our approaches based on IGA work very well for this problem.

\subsubsection{Square plate under biaxial compression}

Finally, we consider a three-layer symmetric cross-ply $\left[0^{0} / 90^{0} / 0^{0}\right]$ simply supported plate subjected to the biaxial buckling load as shown in Fig. 16b. Various length-to-thickness $a / h$ and elastic modulus $E_{1} / E_{2}$ ratios are studied in this example. Table 19 and Table 20 show the normalized critical buckling loads with respect to various modulus and length-to-thickness ratios. The obtained results are compared with those of the finite element formulation based on FSDT [77], the finite element method based on HSDT [69], the meshfree method based on both FSDT and HSDT [67] and also the IGA based on dTrSDTs [38, 7]. The present method shows a very good performance compared to other methods for various modulus ratios and length to thickness ratios. The normalized critical bi-axial buckling loads are increased with respect to increasing the modulus ratio 
Table 18: Normalized critical buckling load of eleven and twenty-one layers sandwich simply supported square plates

\begin{tabular}{|c|c|c|c|c|c|c|}
\hline \multirow[t]{2}{*}{ Layers } & \multirow[t]{2}{*}{$a / h$} & \multirow[t]{2}{*}{ Method } & \multicolumn{4}{|c|}{$h_{f} / h$} \\
\hline & & & 0.025 & 0.050 & 0.075 & 0.1 \\
\hline \multirow[t]{16}{*}{11} & \multirow[t]{8}{*}{10} & FEM-HSDT [46] & 2.2122 & 3.7499 & 4.8643 & 5.7100 \\
\hline & & FEM-FSDT [46] & 2.2043 & 3.8662 & 5.2650 & 6.4930 \\
\hline & & FEM-LW [73] & 2.2592 & 3.7402 & 4.7850 & 5.5618 \\
\hline & & CFS-LW [73] & 2.2639 & 3.7649 & 4.8302 & 5.6255 \\
\hline & & Elasticity [72] & 2.2081 & 3.7385 & 4.8307 & 5.6721 \\
\hline & & IGA (Arya [38]) & 2.3000 & 3.8560 & 4.9554 & 5.7859 \\
\hline & & IGA (Soldatos [7]) & 2.2909 & 3.8335 & 4.9307 & 5.7786 \\
\hline & & IGA (present) & 2.3196 & 3.9091 & 5.0291 & 5.8506 \\
\hline & \multirow[t]{8}{*}{20} & FEM-HSDT [46] & 2.5536 & 4.6756 & 6.4528 & 7.9512 \\
\hline & & FEM-FSDT [46] & 2.5437 & 4.7128 & 6.6156 & 8.2984 \\
\hline & & FEM-LW [73] & 2.5885 & 4.7028 & 6.4604 & 7.9316 \\
\hline & & CFS-LW [73] & 2.566 & 4.6817 & 6.4428 & 7.9184 \\
\hline & & Elasticity [72] & 2.5534 & 4.6460 & 6.4401 & 7.9352 \\
\hline & & IGA (Arya [38]) & 2.5619 & 4.6891 & 6.4624 & 7.9554 \\
\hline & & IGA (Soldatos [7]) & 2.5591 & 4.6807 & 6.4518 & 7.9520 \\
\hline & & IGA (present) & 2.5679 & 4.7085 & 6.4935 & 7.9857 \\
\hline \multirow[t]{16}{*}{21} & \multirow[t]{16}{*}{10} & CSF-HSDT [76] & 2.2621 & 3.7611 & 4.8256 & 5.6215 \\
\hline & & FEM-GLPT [75] & 2.1914 & 3.6770 & 4.7432 & 5.5471 \\
\hline & & CSF-MLW [74] & 2.1904 & 3.6759 & 4.7433 & 5.5463 \\
\hline & & CSF-HSDT [74] & 2.2942 & 3.8475 & 4.9580 & 5.7946 \\
\hline & & Elasticity [72] & 2.2376 & 3.7375 & 4.7637 & 5.6081 \\
\hline & & IGA (Arya [38]) & 2.2993 & 3.8546 & 4.9544 & 5.7869 \\
\hline & & IGA (Soldatos [7]) & 2.2901 & 3.8321 & 4.9300 & 5.7803 \\
\hline & & IGA (present) & 2.3189 & 3.9076 & 5.0277 & 5.8506 \\
\hline & & CSF-HSDT [76] & 2.5658 & 4.6804 & 6.4414 & 7.9171 \\
\hline & & FEM-GLPT [75] & 2.5391 & 4.6387 & 6.3915 & 7.8632 \\
\hline & & CSF-MLW [74] & 2.539 & 4.6386 & 6.3914 & 7.8631 \\
\hline & & CSF-HSDT [74] & 2.6386 & 4.7857 & 6.5644 & 8.0544 \\
\hline & & Elasticity [72] & 2.5543 & 4.6590 & 6.4224 & 7.8969 \\
\hline & & IGA (Arya [38]) & 2.5616 & 4.6884 & 6.4617 & 7.9556 \\
\hline & & IGA (Soldatos [7]) & 2.5588 & 4.6800 & 6.4513 & 7.9527 \\
\hline & & IGA (present) & 2.5677 & 4.7078 & 6.4926 & 7.9853 \\
\hline
\end{tabular}


Table 19: Biaxial critical buckling load of simply supported cross-ply $\left[0^{0} / 90^{0} / 0^{0}\right]$ square plate with various modulus ratios

\begin{tabular}{lllll}
\hline Method & \multicolumn{4}{c}{$E_{1} / E_{2}$} \\
\cline { 2 - 5 } & 10 & 20 & 30 & 40 \\
\hline FEM-FSDT [77] & 4.963 & 7.588 & 8.575 & 10.202 \\
FEM-HSDT [69] & 4.963 & 7.516 & 9.056 & 10.259 \\
IGA (Arya [38]) & 4.9081 & 7.4264 & 8.7790 & 9.9188 \\
IGA (Soldatos [7]) & 4.9092 & 7.4285 & 8.7998 & 9.9495 \\
IGA (present) & 4.9130 & 7.4408 & 8.7550 & 9.8795 \\
\hline
\end{tabular}

Table 20: Biaxial critical buckling load of simply supported cross-ply $\left[0^{0} / 90^{0} / 0^{0}\right]$ square plate with various ratios $a / h$

\begin{tabular}{llllll}
\hline Method & \multicolumn{5}{c}{$a / h$} \\
\cline { 2 - 6 } & 2 & 5 & 10 & 15 & 20 \\
\hline RPIM-HSDT [67] & 1.457 & 5.519 & 10.251 & 12.239 & 13.164 \\
RPIM-FSDT [67] & 1.419 & 5.484 & 10.189 & 12.213 & 13.132 \\
FEM-HSDT [69] & 1.465 & 5.526 & 10.259 & 12.226 & 13.185 \\
IGA (Arya [38]) & 1.3862 & 5.3668 & 9.9188 & 12.0205 & 13.0379 \\
IGA (Soldatos [7]) & 1.3641 & 5.3834 & 9.9495 & 12.0398 & 13.0504 \\
IGA (present) & 1.4316 & 5.3236 & 9.8795 & 11.9978 & 13.0239 \\
\hline
\end{tabular}

$E_{1} / E_{2}$

\section{Conclusions}

An isogeometric finite element formulation in combination with a new inverse tangent shear deformation theory has been proposed for static, free vibration and buckling analysis of laminated composite and sandwich plates. In the present theory, shear stresses free boundary conditions at the top and bottom surfaces of the plates are satisfied and hence shear correction factors are ignored. Weak forms of the static, free vibration and buckling models for laminated composite and sandwich plates using the present theory were derived. Numerical results are presented to investigate the influences of the length to thickness ratio, the core thickness to face sheet thickness ratio, the elastic modulus ratio and various boundary conditions. Obtained results showed high reliability for all test cases from the thin to thick plates. Besides the proposed theory, other trigonometric shear deformation theories $[38,39,7]$ were also considered in this paper. Through all problems tested, obtained results from the IGA based on present theory are more accuracy than the IGA based on dTrSDTs $[38,39,7]$ when compared to the exact elasticity 3D solution.

This paper only restricts to a generalized higher-order shear deformation theory with a fiveparameter displacement field. The present method can be easily extended to other theories proposed in Ambartsumian [3], Reissner [4], Levinson [5], Reddy [6], Karama [8] and Aydogdu [9], etc. This expansion is just simple how to choose the corresponding distribution function $f(z)$ along the plate thickness. Furthermore, the IGA can be applied to the unified framework of the multilayered plates $[16,17]$ and a higher order shear \& normal deformable beam/plate theory (TSNDT) 
$[78,79]$. The authors believe that the present method will be very promising to provide an effectively alternative method of traditional finite elements for analysis of laminated composite and sandwich plate structures in practical applications.

\section{References}

[1] P. Bose and J.N. Reddy. Analysis of composite plates using various plate theories. Part 1. Formulation and analytical solutions. Structural Engineering and Mechanics, 6:583-612, 1998.

[2] J.M. Whitney and N.J. Pagano. Shear deformation in heterogeneous anisotropic plates. Journal of Applied Mechanics, 37(4):1031-1036, 1970.

[3] S.A. Ambartsumian. On the theory of bending plates. Izv Otd Tech Nauk AN SSSR, 5:269$277,1958$.

[4] E. Reissner. On transverse bending of plates including the effects of transverse shear deformation. International Journal of Solids and Structures, 25:495-502, 1975.

[5] M. Levinson. An accurate simple theory of statics and dynamics of elastic plates. Mechanics Research Communications, 7:343350, 1980.

[6] J.N. Reddy. A simple higher-order theory for laminated composite plates. Journal of Applied Mechanics, 51:745-752, 1984.

[7] K.P. Soldatos. A transverse shear deformation theory for homogenous monoclinic plates. Acta Mechanica, 94:195-220, 1992.

[8] M. Karama, K.S. Afaq, and S. Mistou. Mechanical behaviour of laminated composite beam by new multi-layered laminated composite structures model with transverse shear stress continuity. International Journal of Solids and Structures, 40:15251546, 2003.

[9] M. Aydogdu. A new shear deformation theory for laminated composite plates. Composite Structures, 89, 2009.

[10] S.T. Mau. A refined laminate plate theory. Journal of Applied Mechanics, 40:606-607, 1973.

[11] P.C. Chou and J. Corleone. Transverse shear in laminated plate theories. American Institute of Aeronautics and Astronautics, 11:1333-1336, 1973.

[12] M.Di Sciuva. An improved shear-deformation theory for moderately thick multilayered shells and plates. Journal of Applied Mechanics, 54:589-597, 1987.

[13] A. Toledano and H. Murakami. A composite plate theory for arbitrary laminate configuration. Journal of Applied Mechanics, 54:181-189, 1987.

[14] J.G. Ren. A new theory of laminated plate. Composites Science and Technology, 26:225-239, 1986. 
[15] L.M.S. Castro, A.J.M. Ferreira, S. Bertoluzza, R.C. Batra, and J.N.Reddy. A wavelet collocation method for the static analysis of sandwich plates using a layerwise theory, journal = Composite Structures, year $=2010$, volume $=92$, pages $=1786-1792$.

[16] E. Carrera. $C^{0}$ Reissner-Mindlin multilayered plate elements including Zig-Zag and interlaminar stress continuity. International Journal for Numerical Methods in Engineering, 39:1797$1820,1996$.

[17] E. Carrera. Evaluation of layer-wise mixed theories for laminated plate analysis. American Institute of Aeronautics and Astronautics, 36:830-839, 1998.

[18] E. Carrera. Developments, ideas and evaluations based upon the reissners mixed vari- ational theorem in the modeling of multilayered plates and shells. Applied Mechanics Reviews, 54:301-329, 2001.

[19] E. Carrera. Historical review of zig-zag theories for multilayered plates and shells. Applied Mechanics Reviews, 56:287-308, 2003.

[20] T.J.R. Hughes, J.A. Cottrell, and Y. Bazilevs. Isogeometric analysis: CAD, finite elements, NURBS, exact geometry and mesh refinement. Computer Methods in Applied Mechanics and Engineering, 194(39-41):4135-4195, 2005.

[21] J. Cottrell, T.J.R. Hughes, and A. Reali. Studies of refinement and continuity in isogemetric analysis. Computer Methods in Applied Mechanics and Engineering, 196:4160-4183, 2007.

[22] J.A. Cottrell, A. Reali, Y. Bazilevs, and T.J.R. Hughes. Isogeometric analysis of structural vibrations. Computer Methods in Applied Mechanics and Engineering, 195(41-43):52575296, 2006.

[23] T. Elguedj, Y. Bazilevs, V. Calo, and T. Hughes. B and F projection methods for nearly incompressible linear and non-linear elasticity and plasticity using higher-order nurbs elements. Computer Methods in Applied Mechanics and Engineering, 197:27322762, 2008.

[24] W.A. Wall, M.A. Frenzel, and C. Cyron. Isogeometric structural shape optimization. Computer Methods in Applied Mechanics and Engineering, 197(33-40):2976-2988, 2008.

[25] J. Kiendl, K.U. Bletzinger, J. Linhard, and R. Wchner. Isogeometric shell analysis with Kirchhoff-Love elements. Computer Methods in Applied Mechanics and Engineering, 198(49-52):3902-3914, 2009.

[26] J. Kiendl, Y. Bazilevs, M.C. Hsu, R. Wchner, and K.U. Bletzinger. The bending strip method for isogeometric analysis of Kirchhoff-Love shell structures comprised of multiple patches. Computer Methods in Applied Mechanics and Engineering, 199(37-40):2403-2416, 2010.

[27] N. Nguyen-Thanh, J. Kiendl, H. Nguyen-Xuan, R. Wuchner, K.U. Bletzinger, Y. Bazilevs, and T. Rabczuk. Rotation free isogeometric thin shell analysis using PHT-splines. Computer Methods in Applied Mechanics and Engineering, 200(47-48):3410-3424, 2011. 
[28] D.J. Benson, Y. Bazilevs, M.C. Hsu, and T.J.R. Hughes. Isogeometric shell analysis: The Reissner-Mindlin shell. Computer Methods in Applied Mechanics and Engineering, 199(58):276-289, 2010.

[29] Chien H. Thai, H. Nguyen-Xuan, N. Nguyen-Thanh, T-H. Le, T. Nguyen-Thoi, and T. Rabczuk. Static, free vibration, and buckling analysis of laminated composite ReissnerMindlin plates using NURBS-based isogeometric approach. International Journal for Numerical Methods in Engineering, 91:571-603, 2012.

[30] N. Valizadeh, S. Natarajan, O.A. Gonzalez-Estrada, T. Rabczuk, T.Q. Bui, and S. Bordas. NURBS-based finite element analysis of functionally graded plates: Static bending, vibration, buckling and flutter. Composite Structures, 99:309-326, 2013.

[31] H. Kapoor and R.K. Kapania. Geometrically nonlinear nurbs isogeometric finite element analysis of laminated composite plates. Composite Structures, 94:3434-3447, 2012.

[32] Chien H. Thai, H. Nguyen-Xuan, S.P.A. Bordas, N. Nguyen-Thanh, and T. Rabczuk. Isogeometric analysis of laminated composite plates using the higher-order shear deformation theory. Mechanics of Advanced Materials and Structures, accepted, 2012.

[33] Loc V. Tran, A. J. Ferreira, and H. Nguyen-Xuan. Isogeometric approach for analysis of functionally graded plates using higher-order shear deformation theory. Composite Part B, in press, 2013.

[34] D.J. Benson, Y. Bazilevs, M.C. Hsu, and T.J.R. Hughes. A large deformation, rotationfree, isogeometric shell. Computer Methods in Applied Mechanics and Engineering, 200(1316):1367-1378, 2011.

[35] L.A. Piegl, , and W. Tiller. The NURBS book. Springer Verlag, 1997.

[36] J.L. Mantari, A.S. Oktem, and C.G. Soares. A new higher order shear deformation theory for sandwich and composite laminated plates. Composites: Part B, 43:1489-1499, 2012.

[37] N. Grover, D.K. Maiti, and B.N. Singh. A new inverse hyperbolic shear deformation theory for static and buckling analysis of laminated composite and sandwich plates. Composite Structures, 95:667-675, 2013.

[38] H. Arya, R.P. Shimpi, and N.K. Naik. A zigzag model for laminated composite beams. Composite Structures, 56:2124, 2002.

[39] M. Touratier. An efficient standard plate theory. International Journal of Engineering Science, 29:745-752, 1991.

[40] J.N. Reddy. Mechanics of laminated composite plates and shells theory and anlysis, (Second edition). New York: CRC Press, 2004.

[41] F. Auricchio, L. Beirao da Veiga, A. Buffa, C. Lovadina, A. Reali, and G. Sangalli. A fully locking-free isogeometric approach for plane linear elasticity problems: a stream function formulation. Computer Methods in Applied Mechanics and Engineering, 197:160-172, 2007. 
[42] T. Kant and K. Swaminathan. Analysis solutions for the static analysis of laminated composite and sandwich plates based on a higher order refined theory. Composite Structures, 56:329-344, 2002.

[43] K.M. Liew, Y.Q. Huang, and J.N. Reddy. Vibration analysis of symmetrically laminated plates based on FSDT using the moving least squares differential quadrature method. Computer Methods in Applied Mechanics and Engineering, 192:2203-22, 2003.

[44] T. Kant and K. Swaminathan. Analysis solutions for free vibration of laminated composite and sandwich plates based on a higher order refined theory. Composite Structures, 53:73-85, 2001.

[45] X.L. Chen, G.R. Liu, and S.P. Lim. An element free galerkin method for the free vibration analysis of composite laminates of complicated shape. Composite Structures, 59:279-289, 2003.

[46] B.C. Sarah and T. Kant. Two shear deformable finite element models for buckling analysis of skew fiber-reinforced composite and sandwich panels. Composite Structures, 46:115-124, 1999.

[47] N.J. Pagano. Exact solutions for rectangular bidirectional composites and sandwich plates. Journal of Composite Materials, 4:20-34, 1970.

[48] G. Akhras, M.S. Cheung, and W. Li. Finite strip analysis for anisotropic laminated composite plates using higher-order deformation theory. Computers and Structures, 52(3):471-7, 1994.

[49] A.J.M. Ferreira, C.M.C. Roque, and P.A.L.S. Martins. Analysis of composite plates using higher-order shear deformation theory and a finite point formulation based on the multiquadric radial basis function method. Composites: Part B, 34:627-636, 2003.

[50] J.L. Mantari, A.S. Oktem, and C.G. Soares. A new higher order shear deformation theory for sandwich and composite laminated plates. Composites: Part B, (43):1489-1499, 2012.

[51] M. Karama, K.S. Afaq, and S. Mistou. A new theory for laminated composite plates. In: Proc. IMechE (Part L: Journal of Materials: Design and Applications), 223, 2009.

[52] N. Grover, D.K. Maiti, and B.N. Singh. A new inverse hyperbolic shear deformation theory for static and buckling analysis of laminated composite and sandwich plates. Composite Structures, 95, 2013.

[53] S.Srinivas. A refined analysis of composite laminates. Journal of Sound and Vibration, 30:495-507, 1973.

[54] B.N. Pandya and T. Kant. Higher-order shear deformable theories for flexure of sandwich plates-finite element evaluations. International Journal of Solids and Structures, 24:419451, 1988.

[55] A.J.M. Ferreira. Analysis of composite plates using a layerwise theory and multiquadrics discretization. Mechanics of Advanced Materials and Structures, 12:99-112, 2005. 
[56] A.J.M. Ferreira, G.E. Fasshauer, R.C. Batra, and J.D. Rodrigues. Static deformations and vibration analysis of composite and sandwich plates using a layerwise theory and RBF-PS discretizations with optimal shape parameter. Composite Structures, 86:328-343, 2008.

[57] A. Kdheir. Analysis of symmetric cross-ply elastic plates using a higher-order theory, part II: buckling and free vibration. Composite Structures, 9:259-77, 1988.

[58] J.N. Reddy. Mechanics of laminated composite plates. New York: CRC Press, 1997.

[59] A.J.M. Ferreira. A formulation of the multiquadric radial basis function method for the analysis of laminated composite plates. Composite Structures, 59(3):385-92, 2003.

[60] A.J.M. Ferreira, L.M.S. Castro, and S. Bertoluzza. A high order collocation method for the static and vibration analysis of composite plates using a first-order theory. Composite Structures, 89(3):424-432, 2009.

[61] W. Zhen and C. Wanji. Free vibration of laminated composite and sandwich plates using global-local higher-order theory. Journal of Sound and Vibration, 298:333-49, 2006.

[62] C.P. Wu and W.Y. Chen. Vibration and stability of laminated plates based on a local higherorder plate theory. Journal of Sound and Vibration, 177:503-520, 1994.

[63] H. Matsunaga. Vibration and stability of cross-ply laminated composite plates according to a global higher-order plate theory. Composite Structures, 48:231-244, 2000.

[64] K.N. Cho, C.W. Bert, and A.G. Striz. Free vibration of laminated rectangular plates analyzed by higher-order individual-layer theory. Journal of Sound and Vibration, 145:429-442, 1991.

[65] T. Kant and K. Swaminathan. Free vibration of isotropic, orthotropic, and multilayer plates based on higher order refined theories. Journal of Sound and Vibration, 241:319-327, 2001.

[66] A.K. Noor and Mathers. Shear-flexible finite element method of laminated composite plate. Technical report, NASA, 1975.

[67] L. Liu, L.P. Chua, and D.N. Ghista. Mesh-free radial basis function method for static, free vibration and buckling analysis of shear deformable composite laminates. Composite Structures, 78:58-69, 2007.

[68] N.D. Phan and J.N. Reddy. Analysis of laminated composite plates using a higher-order shear deformation theory. International Journal for Numerical Methods in Engineering, 21:2201$2219,1985$.

[69] A.A. Khdeir and L. Librescu. Analysis of symmetric cross-ply elastic plates using a higherorder theory: Part II: buckling and free vibration. Composite Structures, 9:259-277, 1988.

[70] A. Chakrabarti and A.H. Sheikh. Buckling of laminated composite plates by a new element based on higher order shear deformation theory. Mechanics of Composite Materials and Structures, 10(4):303-317, 2003. 
[71] J.N. Reddy and N.D. Phan. Stability and vibration of isotropic, orthotropic and laminated plates according to a higher order shear deformation theory. Journal of Sound and Vibration, 89:157-170, 1985.

[72] A.K. Noor, J.M. Peters, and W.S. Burton. Three-dimensional solutions for initially stressed structural sandwiches. Journal of Engineering Mechanics (ASCE), 120:284-303, 1994.

[73] M. Cetkovic and D. Vuksanovic. Bending, free vibrations and buckling of laminated composite and sandwich plates using a layerwise displacement model. Composite Structures, 88:219-227, 2009.

[74] J.B. Dafedar, Y.M. Desai, and A.A. Mufti. Stability of sandwich plates by mixed, higherorder analytical formulation. International Journal of Solids and Structures, 40:4501-4517, 2003.

[75] M. Shariyat. A generalized high-order globale local plate theory for nonlinear bending and buckling analyses of imperfect sandwich plates subjected to thermo-mechanical loads. Composite Structures, 92:130-143, 2010.

[76] M.M. Kheirikhaha, S.M.R. Khalili, and K.M. Fard. Biaxial buckling analysis of soft-core composite sandwich plates using improved high-order theory. European Journal of Mechanics A/Solids, 31:54-66, 2012.

[77] M.E. Fares and A.M. Zenkour. Buckling and free vibration of non-homogeneous composite cross-ply laminated plates with various plate theories. Composite Structures, 44:279-287, 1999.

[78] J.R. Xiao, D.F. Gilhooley, R.C. Batra, J.W. Gillespie Jr., and M.A. McCarthy. Analysis of thick composite laminates using a higher-order shear and normal deformable plate theory (HOSNDPT) and a Meshless Method. Composite Part B, 39:414-427, 2008.

[79] R.C. Batra and J. Xiao. Venant-Kirchhoff beam using layer-wise third order shear and normal deformable beam theory (TSNDT). Composite Structures, 97:147-1614, 2013. 